\title{
Radio sources in low-luminosity active galactic nuclei
}

\section{Radio luminosity function, importance of jet power, and radio properties of the complete Palomar sample}

\author{
N. M. Nagar ${ }^{1,2}$, H. Falcke ${ }^{3}$, and A. S. Wilson ${ }^{4}$ \\ 1 Kapteyn Institute, Landleven 12, 9747 AD Groningen, The Netherlands \\ 2 Astronomy Group, Departamento de Física, Universidad de Concepción, Casilla 160-C, Concepción, Chile \\ e-mail: nagar@astro-udec.cl \\ 3 ASTRON, PO Box 2, 7990 AA Dwingeloo, The Netherlands \\ Department of Astronomy, Radboud University Nijmegen, Postbus 9010, 6500 GL Nijmegen, The Netherlands \\ e-mail: falcke@astron.nl \\ 4 Department of Astronomy, University of Maryland, College Park, MD 20742, USA \\ Adjunct Astronomer, Space Telescope Science Institute, 3700 San Martin Drive, Baltimore, MD 21218, USA \\ e-mail: wilson@astro.umd.edu
}

Received 29 October 2004 / Accepted 5 February 2005

\begin{abstract}
We present the completed results of a high resolution radio imaging survey of all ( 200) low-luminosity active galactic nuclei (LLAGNs) and AGNs in the Palomar Spectroscopic Sample of all ( 488) bright northern galaxies. The high incidences of pc-scale radio nuclei, with implied brightness temperatures $\gtrsim 10^{7} \mathrm{~K}$, and sub-parsec jets argue for accreting black holes in $\gtrsim 50 \%$ of all LINERs and low-luminosity Seyferts; there is no evidence against all LLAGNs being mini-AGNs. The detected parsec-scale radio nuclei are preferentially found in massive ellipticals and in type 1 nuclei (i.e. nuclei with broad $\mathrm{H} \alpha$ emission). The radio luminosity function (RLF) of Palomar Sample LLAGNs and AGNs extends three orders of magnitude below, and is continuous with, that of "classical" AGNs. We find marginal evidence for a low-luminosity turnover in the RLF; nevertheless LLAGNs are responsible for a significant fraction of present day mass accretion. Adopting a model of a relativistic jet from Falcke \& Biermann, we show that the accretion power output in LLAGNs is dominated by the kinetic power in the observed jets rather than the radiated bolometric luminosity. The Palomar LLAGNs and AGNs follow the same scaling between jet kinetic power and narrow line region (NLR) luminosity as the parsec to kilo-parsec jets in powerful radio galaxies. Eddington ratios $l_{\text {Edd }}\left(=L_{\text {Emitted }} / L_{\text {Eddington }}\right)$ of $\leq 10^{-1}-10^{-5}$ are implied in jet models of the radio emission. We find evidence that, in analogy to Galactic black hole candidates, LINERs are in a "low/hard" state (gas poor nuclei, low Eddington ratio, ability to launch collimated jets) while low-luminosity Seyferts are in a "high" state (gas rich nuclei, higher Eddington ratio, less likely to launch collimated jets). In addition to dominating the radiated bolometric luminosity of the nucleus, the radio jets are energetically more significant than supernovae in the host galaxies, and are potentially able to deposit sufficient energy into the innermost parsecs to significantly slow the gas supply to the accretion disk.
\end{abstract}

Key words. accretion, accretion disks - galaxies: jets - galaxies: nuclei - radio continuum: galaxies - surveys

\section{Introduction}

\subsection{Accretion activity: Ubiquity and scaling}

It is now clear that there is no sharp division between "active" galactic nuclei (AGN; i.e. nuclei presumably powered by accretion onto a nuclear supermassive ( $\gg 10^{5} M_{\odot}$ ) black hole) and "inactive" or "normal" galactic nuclei (nuclei powered by star-formation-related processes). Rather, there is a continuous

* Tables 1 and 2 are also available in electronic form at the CDS via anonymous ftp to cdsarc.u-strasbg.fr $(130.79 .128 .5)$ or via http://cdsweb.u-strasbg.fr/cgi-bin/qcat?J/A+A/435/521 sequence of activity levels between these two extremes. There are two lines of evidence for this continuity. The first comprises the ubiquity of black holes and the correlations between black hole mass, galaxy bulge mass and galaxy bulge velocity dispersion (Richstone et al. 1998; Ferrarese \& Merritt 2000; Gebhardt et al. 2000; Merritt \& Ferrarese 2001; Tremaine et al. 2002; Marconi \& Hunt 2003). These results support the idea that many galactic nuclei are quasar relics (Soltan 1982) and highlight the importance of studying the coeval evolution of a galaxy and its nuclear black hole. The second line of evidence is that many nearby galaxy nuclei not considered to be powerful AGNs, show several characteristics in common 
with powerful AGNs. These similarities include the presence of compact radio nuclei and sub-parsec to $100 \mathrm{pc}$-scale radio jets (e.g. Heckman 1980; Nagar et al. 2002a), emission line ratios characteristic of powerful AGNs (e.g. Heckman 1980; Ho et al. 1997a), broad $\mathrm{H} \alpha$ lines (Ho et al. 1997b), broader $\mathrm{H} \alpha$ lines in polarized emission than in total emission (Barth et al. 1999), water vapor megamasers (Braatz et al. 1997), and nuclear point-like UV sources (Maoz et al. 1995; Barth et al. 1998).

Important results on the growth of galaxies and their black holes, and on the properties and history of accretion in AGNs, are now being provided by several large surveys, e.g. the Sloan Digital Sky Survey (SDSS; Stoughton et al. 2002). An important complement to these large (and higher redshift) surveys of AGNs is the study of so-called low-luminosity AGNs (LLAGNs; i.e. low-luminosity Seyferts, LINERs, and "transition" nuclei (nuclei with spectra intermediate between those of LINERs and $\mathrm{H}$ II regions)). Here we use the term LLAGN in a more agnostic manner than AGN: we assume that AGNs are powered by accretion onto a supermassive black hole but make no a priori assumption about the power source of LLAGNs. The emission-line luminosities of LLAGNs $\left(L_{\mathrm{H} \alpha} \leq\right.$ $10^{40} \mathrm{erg} \mathrm{s}^{-1}$ by definition; Ho et al. 1997a) are a factor $\sim 10^{2}$ times weaker than typical SDSS AGNs. If LLAGNs are truly (weak) AGNs, then extending our studies to LLAGNs is important as they greatly outnumber powerful AGNs. LLAGNs are best studied in close $(\$ 30 \mathrm{Mpc})$ nuclei, as a result of sensitivity limitations and the need to attain adequate linear resolution to separate any weak accretion related emission from that of the bright host galaxy. In this paper we focus on the radio properties of the $\sim 200$ LLAGNs and AGNs (median distance $\sim 17 \mathrm{Mpc}$ ) in the Palomar spectroscopic sample of $\sim 488$ bright northern galaxies (Ho et al. 1997a). The weak emissionlines of the Palomar LLAGNs can be modeled in terms of photoionization by hot, young stars (Terlevich \& Melnick 1985; Filippenko \& Terlevich 1992; Shields 1992), by collisional ionization in shocks (Koski \& Osterbrock 1976; Fosbury et al. 1978; Heckman 1980; Dopita \& Sutherland 1995) or by starbursts (Alonso-Herrero et al. 1999). Alternatively, they could trace AGNs accreting either at very low accretion rates (with radiated luminosity as low as $\sim 10^{-2}-10^{-7}$ of the Eddington Luminosity, $L_{\text {Eddington }}$ ), or at radiative efficiencies (the ratio of radiated energy to accreted mass) much lower than the typical value of $\sim 10 \%$ (e.g. Chap. 7.8 of Frank et al. 1995) assumed for powerful AGNs.

Closely related to these theoretical and observational studies of the radiation from LLAGNs is the increasing number of accurate mass determinations for "massive dark objects", presumably black holes, in nearby galactic nuclei, as measured directly by kinematics (e.g. Gebhardt et al. 2003) or inferred via the correlation between black hole mass $\left(M_{\mathrm{BH}}\right)$ and central stellar velocity dispersion $\left(\sigma_{\mathrm{c}}\right.$; Ferrarese \& Merritt 2000; Gebhardt et al. 2000; Merritt \& Ferrarese 2001; Tremaine et al. 2002) or galaxy bulge mass (Richstone et al. 1998; Marconi $\&$ Hunt 2003). These mass determinations, coupled with the emitted luminosity from the AGN, enable a measure of the Eddington ratio, i.e. the emitted accretion luminosity in units of the Eddington luminosity $\left(l_{\text {Edd }}=L_{\text {Emitted }} / L_{\text {Eddington }}\right)$. In this paper we argue that accounting for the kinetic power in the radio jet is crucial when estimating $L_{\text {Emitted }}$ (and hence $l_{\text {Edd }}$ ) in LLAGNs even though the radiated luminosity in the radio band is bolometrically unimportant. Our high resolution radio observations of a large number of nearby LLAGNs considerably increase the number of LLAGNs with reliable black hole mass estimates and high resolution radio observations, allowing a better test of the relationship between these quantities.

\subsection{Identifying weak $A G N s$ via their radio emission}

As discussed in Sect. 1 of Nagar et al. (2002a), a sub-parsec, high brightness temperature $\left(T_{\mathrm{b}} \gtrsim 10^{7} \mathrm{~K}\right)$, flat spectrum nuclear radio source and any radio jets are reliable indicators of the presence of an accreting supermassive black hole. Interestingly, compact flat spectrum radio nuclei are also detected toward the $\sim 10-15 M_{\odot}$ black holes in Galactic X-ray binary sources, especially during phases of highly sub-Eddington accretion (see review by Fender \& Belloni 2004). The only known sources of $\log \left[T_{\mathrm{b}}(\mathrm{K})\right] \geq 5$ radio emission in compact starbursts are radio supernovae ( $\mathrm{RSNe}$ ) within the starburst (e.g. Condon et al. 1991; Smith et al. 1998). However, even RSNe or groups of RSNe cannot reproduce the compactness, high brightness temperatures, and flat spectral indices seen in the radio nuclei of LLAGNs (discussed in Sect. 6). It then only remains to use the radio morphology and spectral shape to test whether the radio emission originates from a jet (possibly relativistic) launched by the black hole or from the accretion flow itself. Radio emission is expected from radiatively inefficient accretion flows (RIAFs), e.g. advection-dominated (ADAF; Narayan et al. 1998) or convection-dominated (CDAF; Narayan et al. 2000) accretion flows, possible forms of accretion onto a black hole at low accretion rates (Rees et al. 1982).

We have argued (Nagar et al. 2002a) that the combination of the Very Large Array ${ }^{1}$ (VLA; Thompson et al. 1980) and the Very Long Baseline Array ${ }^{1}$ (VLBA; Napier et al. 1994) makes a highly effective and efficient tool to unambiguously identify weak AGNs in bright galactic nuclei. The main advantages are the minimal obscuration at high gigahertz frequencies, the high resolution which allows one to easily pick out the AGN as most radio emission from other sources is usually resolved out, and the high sensitivity.

\subsection{Previous and future work in this series}

The effectiveness of radio searches for AGNs in LLAGNs is borne out by the results of previous papers in this series. These include results of VLA and VLBA observations of 48 LINERs (Nagar et al. 2000; Falcke et al. 2000, Papers I and II), and a distance-limited ( $D \leq 19 \mathrm{Mpc}$ ) sample of 96 LLAGNs (Nagar et al. 2002a, Paper III), from the Palomar Sample. These papers showed that $250 \%$ of all LINERs and low-luminosity Seyferts have compact flat-spectrum radio nuclei at 150 mas resolution. Follow-up VLBA imaging attained a 100\% detection rate of high brightness temperature milli-arcsec scale nuclei in a radio-flux limited subsample. The compactness, high

1 The VLA and VLBA are operated by the National Radio Astronomy Observatory, a facility of the National Science Foundation operated under cooperative agreement by Associated Universities, Inc. 
brightness-temperature $\left(\geq 10^{6} \mathrm{~K}\right)$, and other properties, all argue for an origin of the radio emission in AGN-related processes. The morphology (sub-parsec jets are detected in several nuclei) and radio spectral shape (Nagar et al. 2001, 2002b) support the dominant source of radio emission as the self-absorbed base of a relativistic jet launched by the black hole, rather than a radiatively inefficient accretion inflow. Compact radio nuclei are preferentially found in massive ellipticals and in type $1 \mathrm{nu}-$ clei. The core radio luminosity is correlated with the nuclear optical "broad" $\mathrm{H} \alpha$ luminosity, the nuclear optical "narrow" emission-line luminosity and width, and the galaxy luminosity (Nagar et al. 2002a). In these correlations, LLAGNs fall close to the low-luminosity extrapolations of more powerful AGNs. The sub-arcsec radio luminosity is correlated with both the estimated mass of the nuclear black hole and the galaxy bulge luminosity. Partial correlation analysis on the two correlations yields the result that each correlation is meaningful even after removing the effect of the other correlation (Nagar et al. 2002a).

This paper presents completed results of our high resolution radio imaging survey of all LLAGNs and AGNs in the Palomar Sample. Future papers will present results on the $1.4 \mathrm{GHz}$ to $667 \mathrm{GHz}$ radio spectral shapes of a subsample of LLAGNs (Nagar et al., in prep.; preliminary results in Nagar et al. 2002b) and the sub-pc jet morphology and jet proper motions in LLAGNs (Nagar et al., in prep.).

\subsection{Organization of this paper}

In the following sections, we first define the sample used (Sect. 2) and then summarize previous observations and report on new VLA and VLBA observations which complete the radio survey of LLAGNs and AGNs in the Palomar sample (Sect. 3). The results of all the VLA and VLBA radio observations of the Palomar sample are presented in Sect. 4. These results are used to synthesize an overall picture of the incidence and properties of AGNs in LLAGNs - including the radio luminosity function, importance of jet energetics, and correlations with other emission-line and host galaxy properties - and their continuity with more powerful AGNs (Sect. 5). The results are briefly discussed in Sect. 6 and the major conclusions of the completed radio study are listed in Sect. 7. Finally, the appendix contains a compilation of high resolution radio observations of the 53 absorption-line nuclei in the Palomar sample. In this paper, as in previous papers of this series, we use a Hubble constant $H_{0}=75 \mathrm{~km} \mathrm{~s}^{-1} \mathrm{Mpc}^{-1}$ to be consistent with Ho et al. (1997a) who tabulate the results of optical spectroscopy of the Palomar sample. In this paper we use "radio luminosity" to denote the radiated power at a given radio frequency and "jet power" or "jet kinetic power" to denote the kinetic or mechanical power in the jet (as derived from models of relativistic jets).

\section{Sample}

The results in this paper, and the new observations reported here, are based on LLAGNs and AGNs selected from the Palomar spectroscopic survey of all ( 488) northern galaxies with $B_{\mathrm{T}}<12.5 \mathrm{mag}$ (Ho et al. 1995). Spectroscopic parameters (including activity classification) of 418 galaxies in the Palomar spectroscopic survey which show nuclear emission lines have been presented in Ho et al. (1997a); updates to these, and upper limits to the emission-line fluxes of a further $53 \mathrm{nu}-$ clei without detected emission-lines, are presented in Ho et al. (2003a). Of the 418 galaxies with nuclear emission lines in Ho et al. (1997a), we consider only the 403 which belong to the defined Palomar sample. We thus included in our radio survey approximately 7 AGNs and 190LLAGNs (using the operational boundary of $L_{\mathrm{H} \alpha} \leq 10^{40} \mathrm{erg} \mathrm{s}^{-1}$ to distinguish LLAGNs from AGNs: Ho et al. 1997a). The 206 nuclei with H II region type spectra which make up the balance of the 403 are excluded from our survey. Of the 7 AGNs, only two (NGC 1275 and NGC 4151) have $\mathrm{H} \alpha$ luminosities significantly greater than the boundary between AGNs and LLAGNs. The other five AGNs are within a factor $\sim 2$ of the boundary. In view of the significant photometric uncertainties (for some of these nuclei) and the large aperture of the $\mathrm{H} \alpha$ luminosity measurements, these 5 AGNs could be loosely considered as LLAGNs. Thus, while we use "Palomar LLAGNs and AGNs" to describe the sample observed in the radio, it is worth bearing in mind that this sample is almost exclusively comprised of LLAGNs. We have not observed the 53 nuclei without detected emission lines (Ho et al. 2003a), but list their radio flux densities, derived from the literature, in the Appendix.

\section{Radio observations of the Palomar sample}

Several earlier surveys have targeted a substantial fraction of the nearby galaxies which are now in the Palomar Sample. Radio surveys with resolution $\sim 1^{\prime \prime}-10^{\prime \prime}$ include those by Heckman et al. (1980); Hummel (1980); Wrobel \& Heeschen (1984); Hummel et al. (1987); Fabbiano et al. (1989); Carral et al. (1990); Wrobel \& Heeschen (1991); Laurent-Muehleisen et al. (1997) and Wrobel et al. (2004). Higher resolution surveys (VLBA or VLBI) include those by Jones et al. (1981) and Hummel et al. (1982).

Since the publishing of comprehensive optical results on the Palomar Sample, three groups have conducted large radio surveys of the sample. Our group has now completed a 0 ' 15 resolution $15 \mathrm{GHz}(2 \mathrm{~cm})$ VLA survey of all LLAGNs except some transition nuclei at $D>19 \mathrm{Mpc}$ (a total of 162 nuclei observed; Nagar et al. 2000, 2002a, this work). We then observed all strong sources with the VLBA unless they had already been observed at VLBI resolution (Falcke et al. 2000; Nagar et al. 2002a, this work). Ho \& Ulvestad (2001) and Ulvestad \& Ho (2001a) have observed all (45) Palomar Seyferts at arcsec resolution at $5 \mathrm{GHz}(6 \mathrm{~cm})$ and $1.4 \mathrm{GHz}(20 \mathrm{~cm})$ and followed up the strong detections at multiple frequencies with the VLBA (Anderson et al. 2004). Filho et al. (2000) and Filho et al. (2004) have completed a 5" $-0{ }^{\prime} 3$ resolution survey of all transition nuclei in the sample with follow up VLBA observations of some of the stronger nuclei. Finally, Ulvestad \& Ho (2001b) have completed a survey of a well-defined sub-sample of $40 \mathrm{H}$ II type nuclei in the Palomar sample and found that none of them has a compact radio nucleus at the flux levels of those in LLAGNs in the sample. The latter result justifies the 
exclusion of $\mathrm{H}$ II type nuclei from the remaining discussion of this paper.

With the new VLA observations reported here, all except 4 of the LLAGNs and AGNs in the Palomar sample have now been observed at sub-arcsec resolution with the VLA. The four exceptions are: NGC 5850, NGC 5970, NGC 5982, and NGC 5985. We believe that none of these four nuclei would have been detected in our survey, since their measured fluxes are $<1 \mathrm{mJy}$ in observations at $1.4-5 \mathrm{GHz}$ with $1^{\prime \prime}-5^{\prime \prime}$ resolution (Hummel et al. 1987; Wrobel \& Heeschen 1991). With the new VLBA observations reported here, all Palomar LLAGNs and AGNs with $S_{15 \mathrm{GHz}}^{\mathrm{VLA}}>2.7 \mathrm{mJy}$ (except NGC 5377) have been observed at milli-arcsecond resolution with the VLBA.

\subsection{New VLA observations and data reduction}

Fifty one Seyferts and LINERs at $D>19 \mathrm{Mpc}$ were observed at $15 \mathrm{GHz}(2 \mathrm{~cm})$ with the VLA in a 14 hour run on 2001 January 13 and 14. The VLA was in A-configuration (Thompson et al. 1980) at this time and was configured to observe in full polarization mode with two channels ("IF"s) of $50 \mathrm{MHz}$ each. Most target sources were observed at elevations between $40^{\circ}$ and $60^{\circ}$; only a few southern sources were observed at lower elevations, but always above $33^{\circ}$. Each target source was observed with a 7 min integration sandwiched between two 1 min observations on a nearby strong point-like source (the "phase calibrator"). Typically, each target galaxy was observed once in this way; for a handful of galaxies, we were able to make two such passes. The following galaxies not in the Palomar sample, but selected for having accurate black hole mass measurements (Richstone et al. 1998), were also observed during the run: NGC 205, NGC 221, NGC 821, NGC 1023, NGC 2300, NGC 7332, NGC 7457, and NGC 7768.

Data were calibrated and mapped using AIPS, following the standard reduction procedures as outlined in the AIPS cookbook $^{2}$. Elevation dependent effects were removed using the post-October 2000 antenna gain solutions, along with corrections for the sky opacity during the run. Observations of 3C 286 (observed at elevation $50^{\circ}$ ) were used to set the fluxdensity scale at $15 \mathrm{GHz}$. A second flux calibrator, 0410+769 (a.k.a. 0409+768; observed at elevation $47^{\circ}$ ), was also observed as a flux check source. The $1 \sigma$ error in flux bootstrapping (i.e. setting the flux density scale relative to the flux calibrators) is expected to be roughly $2.5 \%$.

Maps in Stokes I were made with task IMAGR. Since most targets were observed in "snapshot mode" (i.e. for a short period at a single hour angle), the synthesized beam was not optimal, some of the maps have a low signal-to-noise ratio and any extended structure would not have been mapped properly. For sources stronger than about $3 \mathrm{mJy}$, we were able to iteratively self-calibrate the data so as to increase the signal-tonoise ratio in the final map. The total flux of the source did not change appreciably during this self calibration process; we therefore did not scale up (to compensate for errors in the phase calibration process) the fluxes of sources weaker than $3 \mathrm{mJy}$.

\footnotetext{
2 Available online at www.nrao.edu
}

The resolution of the final maps was $\sim 0.15$. The typical root mean square (rms) noise in the final maps was $0.3 \mathrm{mJy}$ and we use a formal detection limit of $1.5 \mathrm{mJy}$ (i.e. $5 \sigma$ ). However, weaker sources (down to $0.7 \mathrm{mJy}$ ) have been tentatively detected in some nuclei with positions coincident with the highly accurate positions of the optical nuclei (Cotton et al. 1999).

\subsection{New VLBA observations and data reduction}

In order to obtain uniform mas-resolution maps of all LLAGNs and AGNs in the Palomar sample with $S_{15 \mathrm{GHz}}^{\mathrm{VLA}}>2.7 \mathrm{mJy}$, we selected 10LLAGNs from Table 1 which had not previously been observed at high enough resolution, signal-tonoise, or image fidelity with the VLBA or VLBI at $5 \mathrm{GHz}$. These ten LLAGNs, plus one galaxy with an H II type nucleus (NGC 3690), were observed with the VLBA at $4.9 \mathrm{GHz}(6 \mathrm{~cm})$ in a 15 hour run on December 17, 2001.

All observations were performed in single polarization ("LL") mode, with 128 Megabits per second bitrate, and with 4 channels ("BB"s) of bandwidth $8 \mathrm{MHz}$ each. Each source was observed at two or three different hour angles in order to obtain good $(u, v)$-coverage. For each source, the first observation pass used a cycle of $4 \mathrm{~min}$ on source and $1 \mathrm{~min}$ on a nearby (distance $\left.1.5^{\circ}-5.5^{\circ}\right)$ phase calibrator, repeated seven times. The second pass used a cycle of 2 min on source and $1 \mathrm{~min}$ on a nearby phase calibrator, repeated 13 times. The total integration time on each source was therefore at least (and typically) $54 \mathrm{~min}$. The "fringe finder" sources J0555+3948 and J0927+3902 were briefly observed and later used for first order synchronization of the data from the different antennas. The weather was mostly fair at all VLBA sites. There were a few intermittent tape problems at several antennas; all data points with tape weights less than 0.7 (on a scale of 0 to 1) were deleted. Data were calibrated using AIPS, closely following the procedures in the "VLBA pipeline" (Sjouwerman et al. in prep). Bad $(u, v)$ data were deleted before the phase solutions of the phase-calibrator observations were transfered to the galaxy data.

Images in Stokes I (assuming no circular polarization) of the sources were made using AIPS task IMAGR. For sources stronger than about $3 \mathrm{mJy}$, we were able to iteratively selfcalibrate and image the data so as to increase the signal-tonoise in the final map. The peak flux-density of the source typically increased by a factor of 1.3 during the self-calibration process. Therefore, for sources weaker than $4 \mathrm{mJy}$, on which accurate self-calibration was not possible, we have multiplied the peak detected flux-density by 1.3 as a crude attempt to correct for atmospheric decorrelation losses. The rms noise in the final, uniformly-weighted images is typically $0.15 \mathrm{mJy}$ to $0.2 \mathrm{mJy}$, and the resolution between 2 mas and 5 mas.

\section{Results of the radio observations}

\subsection{Results of the VLA observations}

The detection rate of radio nuclei with the VLA is illustrated in Fig. 1. When all LLAGNs and AGNs in the Palomar sample are considered, the VLA observations have detected 21 of $45(47 \% \pm 10 \%)$ Seyferts, 37 of $84(44 \% \pm 7 \%)$ LINERs, and 10 of $64(16 \% \pm 5 \%)$ transition nuclei, at a resolution of $\sim 0 . ' 15$ and 
Table 1. High-resolution 15 GHZ VLA imaging of Palomar sample AGN and LLAGN.

\begin{tabular}{|c|c|c|c|c|c|c|c|c|c|c|}
\hline Name & $\begin{array}{c}\text { Activity } \\
\text { Type } \\
\text { (2) }\end{array}$ & (3) & $\begin{array}{l}\text { Dist. } \\
\text { (Mpc) } \\
(4)\end{array}$ & $\begin{array}{l}\text { R.A. } \\
\text { (J2000) } \\
(5)\end{array}$ & $\begin{array}{c}\text { Dec. } \\
(\mathrm{J} 2000) \\
(6)\end{array}$ & $\begin{array}{l}\Delta \\
\left({ }^{\prime \prime}\right) \\
(7)\end{array}$ & $\begin{array}{c}\mathrm{S}_{\text {peak }} \\
(\mathrm{mJy} / \\
\text { beam }) \\
(8)\end{array}$ & $\begin{array}{c}\begin{array}{c}S_{\text {tot }} \\
(\mathrm{mJy})\end{array} \\
(9)\end{array}$ & $\begin{array}{c}\log \mathrm{L}_{15 \mathrm{GHz}}^{\text {peak }} \\
\left(\mathrm{W} \mathrm{Hz}^{-1}\right) \\
(10)\end{array}$ & (11) \\
\hline IC 239 & L2:: & 6.0 & 16.8 & $\ldots$ & $\ldots$ & $\ldots$ & $\ldots$ & $<0.9$ & $<19.48$ & \\
\hline IC 356 & $\mathrm{~T} 2$ & 2.0 & 18.1 & $\ldots$ & $\ldots$ & $\ldots$ & $\ldots$ & $<1.0$ & $<19.59$ & 4 \\
\hline IC 520 & $\mathrm{~T} 2$ : & 2.0 & 47.0 & $\ldots$ & $\ldots$ & $\ldots$ & $\ldots$ & $<1.0$ & $<20.42$ & 4 \\
\hline IC 1727 & $\mathrm{~T} 2 / \mathrm{L} 2$ & 9.0 & 8.2 & $\ldots$ & $\ldots$ & $\ldots$ & $\ldots$ & $<0.9$ & $<18.86$ & \\
\hline NGC 185 & S2 & -5.0 & 0.7 & $\ldots$ & $\ldots$ & $\ldots$ & $\ldots$ & $<1.1$ & $<16.81$ & \\
\hline NGC 266 & L1.9 & 2.0 & 62.4 & 004947.819 & 321639.71 & 2.0 & 4.1 & 4.1 & 21.28 & \\
\hline NGC 315 & L1.9 & -4.0 & 65.8 & $a$ & $a$ & $\ldots$ & 470.0 & $\ldots$ & 23.39 & 9 \\
\hline NGC 404 & $\mathrm{~L} 2$ & -3.0 & 2.4 & $\ldots$ & $\ldots$ & $\ldots$ & $\ldots$ & $<1.3$ & $<17.95$ & \\
\hline NGC 410 & $\mathrm{~T} 2:$ & -4.0 & 70.6 & $\ldots$ & $\ldots$ & $\ldots$ & $\ldots$ & $<1.0$ & $<20.78$ & 4 \\
\hline NGC 428 & L2/T2: & 9.0 & 14.9 & $\ldots$ & $\ldots$ & $\ldots$ & $\ldots$ & $<0.9$ & $<19.38$ & \\
\hline NGC 474 & $\mathrm{~L} 2::$ & -2.0 & 32.5 & $\ldots$ & $\ldots$ & $\ldots$ & $\ldots$ & $<1.5$ & $<20.28$ & \\
\hline NGC 488 & $\mathrm{~T} 2::$ & 3.0 & 29.3 & $\ldots$ & $\ldots$ & $\ldots$ & $\ldots$ & $<1.0$ & $<20.01$ & 4 \\
\hline NGC 521 & $\mathrm{~T} 2 / \mathrm{H}:$ & 4.0 & 67.0 & $\ldots$ & $\ldots$ & $\ldots$ & $\ldots$ & $<1.0$ & $<20.73$ & 4 \\
\hline NGC 524 & T2: & -1.0 & 32.1 & $a$ & $a$ & $\ldots$ & 1.5 & 1.5 & 20.27 & 3 \\
\hline NGC 660 & $\mathrm{~T} 2 / \mathrm{H}:$ & 1.0 & 11.8 & $\ldots$ & $\ldots$ & $\ldots$ & $\ldots$ & $<0.9$ & $<19.18$ & \\
\hline NGC 676 & S2: & 0.0 & 19.5 & $\ldots$ & $\ldots$ & $\ldots$ & $\ldots$ & $<1.5$ & $<19.83$ & \\
\hline NGC 718 & L2 & 1.0 & 21.4 & $\ldots$ & $\ldots$ & $\ldots$ & $\ldots$ & $<1.5$ & $<19.91$ & \\
\hline NGC 777 & S2/L2:: & -5.0 & 66.5 & $\ldots$ & $\ldots$ & $\ldots$ & $\ldots$ & $<1.5$ & $<20.90$ & \\
\hline NGC 841 & L1.9: & 2.1 & 59.5 & $\ldots$ & $\ldots$ & $\ldots$ & $\ldots$ & $<1.5$ & $<20.80$ & \\
\hline NGC 1055 & T2/L2:: & 3.0 & 12.6 & $\ldots$ & $\ldots$ & $\ldots$ & $\ldots$ & $<1.8$ & $<19.53$ & \\
\hline NGC 1058 & S2 & 5.0 & 9.1 & $\ldots$ & $\ldots$ & $\ldots$ & $\ldots$ & $<0.9$ & $<18.95$ & \\
\hline NGC 1167 & $\mathrm{~S} 2$ & -3.0 & 65.3 & 030142.347 & 351220.07 & 1.2 & 44.9 & 248.7 & 22.36 & \\
\hline NGC 1169 & L2 & 3.0 & 33.7 & $\ldots$ & $\ldots$ & $\ldots$ & $\ldots$ & $<1.5$ & $<20.31$ & \\
\hline NGC 1275 & S1.5 & 10.0 & 70.1 & $a$ & $a$ & $\ldots$ & 2970.0 & $\ldots$ & 24.24 & 10 \\
\hline NGC 1961 & $\mathrm{~L} 2$ & 5.0 & 53.1 & $\ldots$ & $\ldots$ & $\ldots$ & $\ldots$ & $<1.5$ & $<20.70$ & \\
\hline NGC 2273 & $\mathrm{~S} 2$ & 0.5 & 28.4 & 065008.652 & 605044.95 & 0.8 & 4.1 & 5.1 & 20.60 & \\
\hline NGC 2336 & $\mathrm{~L} 2 / \mathrm{S} 2$ & 4.0 & 33.9 & 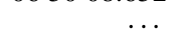 & 年 & $\ldots$ & $x^{2}$ & $<1.5$ & $<20.31$ & \\
\hline NGC 2541 & $\mathrm{~T} 2 / \mathrm{H}:$ & 6.0 & 10.6 & $\ldots$ & $\ldots$ & $\ldots$ & $\ldots$ & $<1.0$ & $<19.13$ & \\
\hline NGC 2655 & S2 & 0.0 & 24.4 & 085538.071 & 781323.77 & 2.8 & 6.0 & 6.0 & 20.63 & \\
\hline NGC 2681 & L1.9 & 0.0 & 13.3 & $\ldots$ & $\ldots$ & $\ldots$ & $\ldots$ & $<1.4$ & $<19.48$ & \\
\hline NGC 2683 & $\mathrm{~L} 2 / \mathrm{S} 2$ & 3.0 & 5.7 & $\ldots$ & $\ldots$ & $\ldots$ & $\ldots$ & $<0.9$ & $<18.54$ & \\
\hline NGC 2685 & $\mathrm{~S} 2 / \mathrm{T} 2:$ & -1.0 & 16.2 & $\ldots$ & $\ldots$ & $\ldots$ & $\ldots$ & $<0.9$ & $<19.45$ & \\
\hline NGC 2768 & L2 & -5.0 & 23.7 & 091137.413 & 600214.86 & 0.9 & 7.9 & 8.2 & 20.73 & \\
\hline NGC 2787 & L1.9 & -1.0 & 13.0 & 091918.607 & 691211.64 & 1.6 & 7.0 & 7.0 & 20.15 & \\
\hline NGC 2832 & $\mathrm{~L} 2::$ & -4.0 & 91.6 & (2) & - T. & 20 & $\ldots$ & $<1.5$ & $<21.18$ & \\
\hline NGC 2841 & $\mathrm{~L} 2$ & 3.0 & 12.0 & 092202.678 & 505835.70 & 7.4 & 1.1 & 2.1 & 19.28 & \\
\hline NGC 2859 & $\mathrm{~T} 2$ & -1.0 & 25.4 & & & $\ldots$ & & $<1.0$ & $<19.89$ & 4 \\
\hline NGC 2911 & $\mathrm{~L} 2$ & -2.0 & 42.2 & 093346.108 & 100908.82 & 0.5 & 17.3 & 17.7 & 21.57 & \\
\hline NGC 2985 & T1.9 & 2.0 & 22.4 & & 10000.02 & $\ldots$ & $\ldots$ & $<1.0$ & $<19.78$ & 4 \\
\hline NGC 3031 & S1.5 & 2.0 & 3.6 & 095533.175 & 690355.06 & 6.4 & 164.1 & 164.8 & 20.41 & \\
\hline NGC 3079 & S2 & 7.0 & 20.4 & a & a & $\ldots$ & 48.0 & 59.0 & 21.38 & 7 \\
\hline NGC 3147 & $\mathrm{~S} 2$ & 4.0 & 40.9 & 101653.648 & 732402.69 & 1.6 & 8.0 & 8.1 & 21.20 & \\
\hline NGC 3166 & L2 & 0.0 & 22.0 & & $\ldots$ & $\ldots$ & $\cdots$ & $<1.5$ & $<19.94$ & \\
\hline NGC 3169 & $\mathrm{~L} 2$ & 1.0 & 19.7 & 101415.052 & 032757.86 & 3.7 & 6.8 & 6.8 & 20.50 & \\
\hline NGC 3190 & $\mathrm{~L} 2$ & 1.0 & 22.4 & 101806.005 & 214954.55 & 5.8 & 1.1 & 0.8 & 19.82 & \\
\hline NGC 3193 & L2: & -5.0 & 23.2 & ... & $\ldots$ & $\ldots$ & $\ldots$ & $<1.5$ & $<19.99$ & \\
\hline NGC 3226 & L1.9 & -5.0 & 23.4 & 102327.014 & 195354.59 & 0.9 & 5.0 & 5.4 & 20.52 & \\
\hline NGC 3227 & S1.5 & 1.0 & 20.6 & 102330.579 & 195154.19 & 0.8 & 3.5 & 4.7 & 20.25 & \\
\hline NGC 3245 & T2: & -2.0 & 22.2 & $\ldots$ & $\ldots$ & $\ldots$ & $\ldots$ & $<1.0$ & $<19.77$ & 4 \\
\hline NGC 3254 & S2 & 4.0 & 23.6 & $\ldots$ & $\ldots$ & $\ldots$ & $\ldots$ & $<1.5$ & $<20.00$ & \\
\hline NGC 3301 & L2 & 0.0 & 23.3 & $\ldots$ & $\ldots$ & $\ldots$ & $\ldots$ & $<1.5$ & $<19.99$ & \\
\hline NGC 3368 & $\mathrm{~L} 2$ & 2.0 & 8.1 & $\ldots$ & $\ldots$ & $\ldots$ & $\ldots$ & $<1.0$ & $<18.89$ & \\
\hline NGC 3379 & L2/T2:: & -5.0 & 8.1 & $\ldots$ & $\ldots$ & $\ldots$ & $\ldots$ & $<1.0$ & $<18.89$ & \\
\hline NGC 3414 & L2 & -2.0 & 24.9 & 105116.210 & 275830.31 & 0.3 & 2.3 & 2.4 & 20.23 & \\
\hline NGC 3433 & L2/T2:: & 5.0 & 39.5 & $\ldots$ & $\ldots$ & $\ldots$ & $\ldots$ & $<1.5$ & $<20.45$ & \\
\hline NGC 3486 & $\mathrm{~S} 2$ & 5.0 & 7.4 & $\ldots$ & $\ldots$ & $\ldots$ & $\ldots$ & $<1.0$ & $<18.82$ & \\
\hline NGC 3489 & $\mathrm{~T} 2 / \mathrm{S} 2$ & -1.0 & 6.4 & $\ldots$ & $\ldots$ & $\ldots$ & $\ldots$ & $<1.0$ & $<18.69$ & \\
\hline NGC 3507 & L2 & 3.0 & 19.8 & $\ldots$ & $\ldots$ & $\ldots$ & $\ldots$ & $<1.5$ & $<19.85$ & \\
\hline NGC 3516 & $\mathrm{~S} 1.2$ & -2.0 & 38.9 & $a$ & $a$ & $\ldots$ & 1.3 & 1.3 & 20.37 & 8 \\
\hline NGC 3607 & L2 & -2.0 & 19.9 & 111654.668 & 180306.43 & 1.2 & 1.6 & 1.4 & 19.88 & \\
\hline NGC 3608 & $\mathrm{~L} 2 / \mathrm{S} 2$ : & -5.0 & 23.4 & $\ldots$ & $\ldots$ & $\ldots$ & $\ldots$ & $<1.5$ & $<19.99$ & \\
\hline NGC 3623 & L2: & 1.0 & 7.3 & $\ldots$ & $\ldots$ & $\ldots$ & $\ldots$ & $<0.9$ & $<18.76$ & \\
\hline NGC 3626 & L2: & -1.0 & 26.3 & $\ldots$ & $\ldots$ & $\ldots$ & $\ldots$ & $<1.5$ & $<20.09$ & \\
\hline NGC 3627 & $\mathrm{~T} 2 / \mathrm{S} 2$ & 3.0 & 6.6 & 112015.008 & 125929.76 & 8.2 & 1.1 & 2.9 & 18.76 & \\
\hline NGC 3628 & $\mathrm{~T} 2$ & 3.0 & 7.7 & 112017.016 & 133520.04 & 6.5 & 1.5 & 2.2 & 19.03 & \\
\hline
\end{tabular}


Table 1. continued.

\begin{tabular}{|c|c|c|c|c|c|c|c|c|c|c|}
\hline Name & $\begin{array}{l}\text { Activity } \\
\text { Type } \\
\text { (2) }\end{array}$ & (3) & $\begin{array}{l}\text { Dist. } \\
\text { (Mpc) } \\
\text { (4) }\end{array}$ & $\begin{array}{l}\text { R.A. } \\
\text { (J2000) } \\
(5)\end{array}$ & $\begin{array}{c}\text { Dec. } \\
(\mathrm{J} 2000) \\
(6)\end{array}$ & $\begin{array}{l}\Delta \\
\left({ }^{\prime \prime}\right) \\
(7)\end{array}$ & $\begin{array}{c}\mathrm{S}_{\text {peak }} \\
(\mathrm{mJy} / \\
\text { beam) } \\
(8)\end{array}$ & $\begin{array}{c}\begin{array}{c}\mathrm{S}_{\mathrm{tot}} \\
(\mathrm{mJy})\end{array} \\
(9)\end{array}$ & $\begin{array}{c}\log \mathrm{L}_{15 \mathrm{GHz}}^{\text {peak }} \\
\left(\mathrm{W} \mathrm{Hz}^{-1}\right) \\
(10)\end{array}$ & Com. \\
\hline NGC 3642 & L1.9 & 4.0 & 27.5 & $\ldots$ & $\ldots$ & $\ldots$ & $\ldots$ & $<1.3$ & $<20.06$ & \\
\hline NGC 3675 & $\mathrm{~T} 2$ & 3.0 & 12.8 & $\ldots$ & $\ldots$ & $\ldots$ & $\ldots$ & $<1.0$ & $<19.29$ & \\
\hline NGC 3681 & $\mathrm{~T} 2:$ & 4.0 & 24.2 & $\ldots$ & $\ldots$ & $\ldots$ & $\ldots$ & $<1.0$ & $<19.85$ & 4 \\
\hline NGC 3692 & $\mathrm{~T} 2$ & 3.0 & 29.8 & $\ldots$ & $\ldots$ & $\ldots$ & $\ldots$ & $<1.2$ & $<20.09$ & \\
\hline NGC 3705 & $\mathrm{~T} 2$ & 2.0 & 17.0 & $\ldots$ & $\ldots$ & $\ldots$ & $\ldots$ & $<1.2$ & $<19.61$ & \\
\hline NGC 3718 & L1.9 & 1.0 & 17.0 & 113234.856 & 530404.51 & 2.0 & 10.5 & 10.8 & 20.56 & \\
\hline NGC 3735 & S2: & 5.0 & 41.0 & $\ldots$ & $\ldots$ & $\ldots$ & $\ldots$ & $<1.5$ & $<20.48$ & \\
\hline NGC 3780 & $\mathrm{~L} 2::$ & 5.0 & 37.2 & 113922.267 & 561610.17 & 4.2 & 1.1 & 1.0 & 20.26 & \\
\hline NGC 3898 & $\mathrm{~T} 2$ & 2.0 & 21.9 & $\ldots$ & $\ldots$ & $\ldots$ & $\ldots$ & $<1.0$ & $<19.76$ & 4 \\
\hline NGC 3900 & L2: & -1.0 & 29.4 & $\ldots$ & $\ldots$ & $\ldots$ & $\ldots$ & $<1.5$ & $<20.19$ & \\
\hline NGC 3917 & $\mathrm{~T} 2:$ & 6.0 & 17.0 & $\ldots$ & $\ldots$ & $\ldots$ & $\ldots$ & $<1.2$ & $<19.63$ & \\
\hline NGC 3941 & S2: & -2.0 & 18.9 & $\ldots$ & $\ldots$ & $\ldots$ & $\ldots$ & $<1.1$ & $<19.67$ & \\
\hline NGC 3945 & $\mathrm{~L} 2$ & -1.0 & 22.5 & 115313.609 & 604032.12 & 1.4 & 1.8 & 2.1 & 20.04 & \\
\hline NGC 3953 & $\mathrm{~T} 2$ & 4.0 & 17.0 & $\ldots$ & $\ldots$ & $\ldots$ & $\ldots$ & $<1.3$ & $<19.64$ & \\
\hline NGC 3976 & $\mathrm{~S} 2$ : & 3.0 & 37.7 & $\ldots$ & $\ldots$ & $\ldots$ & $\ldots$ & $<1.5$ & $<20.41$ & \\
\hline NGC 3982 & S1.9 & 3.0 & 17.0 & $\ldots$ & $\ldots$ & $\ldots$ & $\ldots$ & $<1.0$ & $<19.54$ & \\
\hline NGC 3992 & $\mathrm{~T} 2:$ & 4.0 & 17.0 & $\ldots$ & $\ldots$ & $\ldots$ & $\ldots$ & $<1.3$ & $<19.64$ & \\
\hline NGC 3998 & L1.9 & -2.0 & 21.6 & $a$ & $a$ & $\ldots$ & 57.0 & 57.0 & 21.50 & 12 \\
\hline NGC 4013 & $\mathrm{~T} 2$ & 3.0 & 17.0 & $\ldots$ & $\ldots$ & $\ldots$ & $\ldots$ & $<1.0$ & $<19.54$ & \\
\hline NGC 4036 & L1.9 & -3.0 & 24.6 & 120126.433 & 615345.91 & 2.6 & $\ldots$ & $<1.5$ & $<20.04$ & 2 \\
\hline NGC 4051 & $\mathrm{~S} 1.2$ & 4.0 & 17.0 & $\ldots$ & $\ldots$ & $\ldots$ & $\ldots$ & $<1.0$ & $<19.54$ & \\
\hline NGC 4111 & $\mathrm{~L} 2$ & -1.0 & 17.0 & $\ldots$ & $\ldots$ & $\ldots$ & $\ldots$ & $<1.3$ & $<19.64$ & \\
\hline NGC 4125 & $\mathrm{~T} 2$ & -5.0 & 24.2 & $\ldots$ & $\ldots$ & $\ldots$ & $\ldots$ & $<1.0$ & $<19.85$ & 4 \\
\hline NGC 4138 & S1.9 & -1.0 & 17.0 & 120929.800 & 434106.89 & 1.2 & 1.5 & 1.3 & 19.71 & \\
\hline NGC 4143 & L1.9 & -2.0 & 17.0 & 120936.067 & 423203.05 & 1.6 & 3.3 & 3.3 & 20.06 & \\
\hline NGC 4145 & $\mathrm{~T} 2:$ & 7.0 & 20.7 & $\ldots$ & $\ldots$ & $\ldots$ & $\ldots$ & $<1.3$ & $<19.81$ & \\
\hline NGC 4150 & $\mathrm{~T} 2$ & -2.0 & 9.7 & $\ldots$ & $\ldots$ & $\ldots$ & $\ldots$ & $<1.0$ & $<19.05$ & \\
\hline NGC 4151 & $\mathrm{~S} 1.5$ & 2.0 & 20.3 & $a$ & $a$ & $\ldots$ & 12.5 & 17.6 & 20.79 & 7 \\
\hline NGC 4168 & S1.9: & -5.0 & 16.8 & 121217.270 & 131218.75 & 1.0 & 3.0 & 3.1 & 20.01 & \\
\hline NGC 4169 & S2 & -2.0 & 50.4 & 121219.091 & 291045.03 & 2.3 & 1.2 & 1.0 & 20.56 & \\
\hline NGC 4192 & $\mathrm{~T} 2$ & 2.0 & 16.8 & 121348.269 & 145359.11 & 1.8 & $\ldots$ & $<1.3$ & $<19.64$ & 2 \\
\hline NGC 4203 & L1.9 & -3.0 & 9.7 & 121505.056 & 331150.25 & 1.1 & 9.5 & 9.5 & 20.03 & \\
\hline NGC 4216 & $\mathrm{~T} 2$ & 3.0 & 16.8 & 121554.375 & 130858.13 & 2.2 & 1.2 & 1.3 & 19.61 & \\
\hline NGC 4220 & $\mathrm{~T} 2$ & -1.0 & 17.0 & & & $\ldots$ & $\cdots$ & $<1.4$ & $<19.70$ & \\
\hline NGC 4258 & S1.9 & 4.0 & 6.8 & 121857.505 & 471814.32 & 0.4 & 2.6 & 3.0 & 19.16 & \\
\hline NGC 4261 & $\mathrm{~L} 2$ & -5.0 & 35.1 & $a$ & $a$ & & 300.0 & & 22.65 & 11 \\
\hline NGC 4278 & L1.9 & -5.0 & 9.7 & 122006.824 & 291650.74 & 1.7 & 88.3 & 89.7 & 21.00 & \\
\hline NGC 4281 & $\mathrm{~T} 2::$ & -1.0 & 35.1 & $\ldots$ & $\ldots$ & $\ldots$ & $\ldots$ & $<1.0$ & $<20.17$ & 4 \\
\hline NGC 4293 & $\mathrm{~L} 2$ & 0.0 & 17.0 & 122112.813 & 182256.75 & 5.6 & 0.7 & 1.4 & 19.38 & 6 \\
\hline NGC 4314 & L2 & 1.0 & 9.7 & $\ldots$ & $\ldots$ & $\ldots$ & $\ldots$ & $<1.0$ & $<19.05$ & \\
\hline NGC 4321 & $\mathrm{~T} 2$ & 4.0 & 16.8 & $\ldots$ & $\ldots$ & $\ldots$ & $\ldots$ & $<0.9$ & $<19.48$ & \\
\hline NGC 4324 & $\mathrm{~T} 2::$ & -1.0 & 35.1 & $\ldots$ & $\ldots$ & $\ldots$ & $\ldots$ & $<1.0$ & $<20.17$ & 4 \\
\hline NGC 4346 & $\mathrm{~L} 2::$ & -2.0 & 17.0 & $\ldots$ & $\ldots$ & $\ldots$ & $\ldots$ & $<1.0$ & $<19.54$ & \\
\hline NGC 4350 & $\mathrm{~T} 2::$ & -2.0 & 16.8 & $\ldots$ & $\ldots$ & $\ldots$ & $\ldots$ & $<0.9$ & $<19.48$ & \\
\hline NGC 4374 & L2 & -5.0 & 16.8 & 122503.745 & 125313.19 & 3.2 & 180.7 & 183.7 & 21.79 & \\
\hline NGC 4378 & S2 & 1.0 & 35.1 & $\ldots$ & $\ldots$ & $\ldots$ & $\ldots$ & $<1.5$ & $<20.34$ & \\
\hline NGC 4388 & S1.9 & 3.0 & 16.8 & 122546.783 & 123943.79 & 2.2 & 2.2 & 3.7 & 19.87 & \\
\hline NGC 4394 & L2 & 3.0 & 16.8 & $\ldots$ & $\ldots$ & $\ldots$ & $\ldots$ & $<0.9$ & $<19.48$ & \\
\hline NGC 4395 & $\mathrm{~S} 1.8$ & 9.0 & 3.6 & $\ldots$ & $\ldots$ & $\ldots$ & $\ldots$ & $<0.9$ & $<18.14$ & \\
\hline NGC 4414 & $\mathrm{~T} 2:$ & 5.0 & 9.7 & $\ldots$ & $\ldots$ & $\ldots$ & $\ldots$ & $<0.9$ & $<19.01$ & \\
\hline NGC 4419 & $\mathrm{~T} 2$ & 1.0 & 16.8 & 122656.452 & 150250.80 & 2.8 & 2.7 & 3.6 & 19.96 & \\
\hline NGC 4429 & $\mathrm{~T} 2$ & -1.0 & 16.8 & $\ldots$ & $\ldots$ & $\ldots$ & $\ldots$ & $<1.1$ & $<19.57$ & \\
\hline NGC 4435 & $\mathrm{~T} 2 / \mathrm{H}:$ & -2.0 & 16.8 & $\ldots$ & $\ldots$ & $\ldots$ & $\ldots$ & $<1.1$ & $<19.57$ & \\
\hline NGC 4438 & L1.9 & 0.0 & 16.8 & $\ldots$ & $\ldots$ & $\ldots$ & $\ldots$ & $<0.9$ & $<19.48$ & \\
\hline NGC 4450 & L1.9 & 2.0 & 16.8 & 122829.592 & 170506.01 & 1.4 & 2.0 & 2.7 & 19.83 & \\
\hline NGC 4457 & L2 & 0.0 & 17.4 & $\ldots$ & $\ldots$ & $\ldots$ & $\ldots$ & $<1.0$ & $<19.56$ & \\
\hline NGC 4459 & $\mathrm{~T} 2:$ & -1.0 & 16.8 & $\ldots$ & $\ldots$ & $\ldots$ & $\ldots$ & $<1.0$ & $<19.53$ & \\
\hline NGC 4472 & $\mathrm{~S} 2::$ & -5.0 & 16.8 & 122946.761 & 080001.68 & 1.8 & 3.7 & 4.1 & 20.10 & \\
\hline NGC 4477 & $\mathrm{~S} 2$ & -2.0 & 16.8 & $\ldots$ & $\ldots$ & $\ldots$ & $\ldots$ & $<1.0$ & $<19.53$ & \\
\hline NGC 4486 & L2 & -4.0 & 16.8 & 123049.422 & 122328.03 & 3.1 & 2725.7 & 2835.7 & 22.96 & \\
\hline NGC 4494 & $\mathrm{~L} 2::$ & -5.0 & 9.7 & $\ldots$ & $\ldots$ & $\ldots$ & $\ldots$ & $<0.8$ & $<18.95$ & \\
\hline NGC 4501 & $\mathrm{~S} 2$ & 3.0 & 16.8 & $\ldots$ & $\ldots$ & $\ldots$ & $\ldots$ & $<1.1$ & $<19.57$ & \\
\hline NGC 4527 & $\mathrm{~T} 2$ & 4.0 & 13.5 & $\ldots$ & $\ldots$ & $\ldots$ & $\ldots$ & $<1.1$ & $<19.38$ & \\
\hline NGC 4548 & L2 & 3.0 & 16.8 & 123526.448 & 142946.71 & 2.5 & 1.2 & 1.2 & 19.61 & \\
\hline NGC 4550 & $\mathrm{~L} 2$ & -1.5 & 16.8 & 123530.078 & 121318.63 & 10.8 & 0.7 & 0.7 & 19.37 & 6 \\
\hline
\end{tabular}


Table 1. continued.

\begin{tabular}{|c|c|c|c|c|c|c|c|c|c|c|}
\hline Name & $\begin{array}{l}\text { Activity } \\
\text { Type } \\
\text { (2) }\end{array}$ & (3) & $\begin{array}{c}\text { Dist. } \\
\text { (Mpc) } \\
(4)\end{array}$ & $\begin{array}{c}\text { R.A. } \\
\text { (J2000) } \\
(5)\end{array}$ & $\begin{array}{c}\text { Dec. } \\
\text { (J2000) } \\
(6)\end{array}$ & $\begin{array}{l}\Delta \\
\left({ }^{\prime \prime}\right) \\
(7)\end{array}$ & $\begin{array}{c}\mathrm{S}_{\text {peak }} \\
(\mathrm{mJy} / \\
\text { beam) } \\
(8)\end{array}$ & $\begin{array}{c}\begin{array}{c}\mathrm{S}_{\mathrm{tot}} \\
(\mathrm{mJy})\end{array} \\
(9)\end{array}$ & 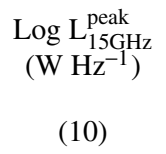 & Com. \\
\hline NGC 4552 & $\mathrm{~T} 2:$ & -5.0 & 16.8 & 123539.807 & 123322.82 & 2.8 & 58.1 & 58.6 & 21.29 & \\
\hline NGC 4565 & S1.9 & 3.0 & 9.7 & 123620.778 & 255915.65 & 4.5 & 3.7 & 3.7 & 19.62 & \\
\hline NGC 4569 & $\mathrm{~T} 2$ & 2.0 & 16.8 & & & & $\ldots$ & $<1.1$ & $<19.57$ & \\
\hline NGC 4579 & S1.9/L1.9 & 3.0 & 16.8 & 123743.523 & 114905.48 & 3.1 & 27.6 & 28.3 & 20.97 & \\
\hline NGC 4589 & $\mathrm{~L} 2$ & -5.0 & 30.0 & 123724.986 & 741130.89 & 1.0 & 11.7 & 11.9 & 21.10 & \\
\hline NGC 4596 & L2:: & -1.0 & 16.8 & ... & $\ldots$ & $\ldots$ & $\ldots$ & $<1.1$ & $<19.57$ & \\
\hline NGC 4636 & L1.9 & -5.0 & 17.0 & 124249.831 & 024115.98 & 2.6 & 1.6 & 1.9 & 19.74 & \\
\hline NGC 4639 & S1.0 & 4.0 & 16.8 & $\ldots$ & $\ldots$ & $\ldots$ & $\ldots$ & $<1.1$ & $<19.57$ & \\
\hline NGC 4643 & $\mathrm{~T} 2$ & 0.0 & 25.7 & $\ldots$ & $\ldots$ & $\ldots$ & $\ldots$ & $<1.0$ & $<19.90$ & 4 \\
\hline NGC 4651 & L2 & 5.0 & 16.8 & $\ldots$ & $\ldots$ & $\ldots$ & $\ldots$ & $<1.1$ & $<19.57$ & \\
\hline NGC 4698 & $\mathrm{~S} 2$ & 2.0 & 16.8 & $\ldots$ & $\ldots$ & $\ldots$ & $\ldots$ & $<1.0$ & $<19.53$ & \\
\hline NGC 4713 & $\mathrm{~T} 2$ & 7.0 & 17.9 & $\ldots$ & $\ldots$ & $\ldots$ & $\ldots$ & $<1.1$ & $<19.63$ & \\
\hline NGC 4725 & S2: & 2.0 & 12.4 & $\ldots$ & $\ldots$ & $\ldots$ & $\ldots$ & $<0.9$ & $<19.22$ & \\
\hline NGC 4736 & L2 & 2.0 & 4.3 & 125053.070 & 410712.95 & 6.2 & 1.9 & 1.7 & 18.62 & \\
\hline NGC 4750 & L1.9 & 2.0 & 26.1 & $\ldots$ & $\ldots$ & $\ldots$ & $\ldots$ & $<1.5$ & $<20.09$ & \\
\hline NGC 4762 & L2: & -2.0 & 16.8 & 125255.903 & 111346.60 & 2.9 & 0.9 & 1.3 & 19.48 & 6 \\
\hline NGC 4772 & L1.9 & 1.0 & 16.3 & 125329.162 & 021006.16 & 2.5 & 3.3 & 3.4 & 20.02 & \\
\hline NGC 4826 & $\mathrm{~T} 2$ & 2.0 & 4.1 & $\ldots$ & $\ldots$ & $\ldots$ & $\ldots$ & $<0.9$ & $<18.26$ & \\
\hline NGC 4866 & L2 & -1.0 & 16.0 & $\ldots$ & $\ldots$ & $\ldots$ & $\ldots$ & $<1.1$ & $<19.53$ & \\
\hline NGC 5005 & L1.9 & 4.0 & 21.3 & $\ldots$ & $\ldots$ & $\ldots$ & $\ldots$ & $<1.1$ & $<19.78$ & \\
\hline NGC 5012 & $\mathrm{~T} 2$ & 5.0 & 40.4 & $\ldots$ & $\ldots$ & $\ldots$ & $\ldots$ & $<1.0$ & $<20.29$ & 4 \\
\hline NGC 5033 & $\mathrm{~S} 1.5$ & 5.0 & 18.7 & 131327.471 & 363537.93 & 1.8 & 1.4 & 1.4 & 19.77 & \\
\hline NGC 5055 & $\mathrm{~T} 2$ & 4.0 & 7.2 & $\ldots$ & $\ldots$ & $\ldots$ & $\ldots$ & $<1.1$ & $<18.83$ & \\
\hline NGC 5194 & $\mathrm{~S} 2$ & 4.0 & 7.7 & $\ldots$ & $\ldots$ & $\ldots$ & $\ldots$ & $<1.1$ & $<18.89$ & \\
\hline NGC 5195 & L2: & 10.0 & 9.3 & $\ldots$ & $\ldots$ & $\ldots$ & $\ldots$ & $<1.1$ & $<19.06$ & \\
\hline NGC 5273 & $\mathrm{~S} 1.5$ & -2.0 & 21.3 & $\ldots$ & .. & $\ldots$ & $\ldots$ & $<1.1$ & $<19.78$ & \\
\hline NGC 5297 & $\mathrm{~L} 2$ & 4.5 & 37.8 & $\ldots$ & $\ldots$ & $\ldots$ & $\ldots$ & $<1.5$ & $<20.41$ & \\
\hline NGC 5322 & $\mathrm{~L} 2::$ & -5.0 & 31.6 & 134915.265 & 601125.93 & 0.0 & 12.7 & 12.7 & 21.18 & \\
\hline NGC 5353 & $\mathrm{~L} 2 / \mathrm{T} 2:$ & -2.0 & 37.8 & 135326.694 & 401658.87 & 0.6 & 18.3 & 18.7 & 21.50 & \\
\hline NGC 5354 & $\mathrm{~T} 2 / \mathrm{L} 2:$ & -2.0 & 32.8 & a & $a$ & & 9.7 & 9.7 & 21.10 & 3 \\
\hline NGC 5363 & $\mathrm{~L} 2$ & 10.0 & 22.4 & 135607.214 & 051517.25 & 2.5 & 38.1 & 40.7 & 21.36 & \\
\hline NGC 5371 & L2 & 4.0 & 37.8 & $\ldots$ & $\ldots$ & $\ldots$ & $\ldots$ & $<1.5$ & $<20.41$ & \\
\hline NGC 5377 & L2 & 1.0 & 31.0 & 135616.834 & 471406.27 & 0.5 & 3.0 & 3.1 & 20.54 & \\
\hline NGC 5395 & $\mathrm{~S} 2 / \mathrm{L} 2$ & 3.0 & 46.7 & $\ldots$ & $\ldots$ & $\ldots$ & $\ldots$ & $<1.5$ & $<20.59$ & \\
\hline NGC 5448 & L2 & 1.0 & 32.6 & $\ldots$ & $\ldots$ & $\ldots$ & $\ldots$ & $<1.5$ & $<20.28$ & \\
\hline NGC 5485 & L2: & -2.0 & 32.8 & $\ldots$ & $\ldots$ & $\ldots$ & $\ldots$ & $<1.5$ & $<20.29$ & \\
\hline NGC 5566 & L2 & 2.0 & 26.4 & $\ldots$ & $\ldots$ & $\ldots$ & $\ldots$ & $<1.1$ & $<19.96$ & \\
\hline NGC 5631 & S2/L2: & -2.0 & 32.7 & $\ldots$ & $\ldots$ & $\ldots$ & $\ldots$ & $<1.5$ & $<20.28$ & \\
\hline NGC 5656 & $\mathrm{~T} 2::$ & 2.0 & 42.6 & $\ldots$ & $\ldots$ & $\ldots$ & $\ldots$ & $<1.0$ & $<20.34$ & 4 \\
\hline NGC 5678 & $\mathrm{~T} 2$ & 3.0 & 35.6 & $\ldots$ & $\ldots$ & $\ldots$ & $\ldots$ & $<1.0$ & $<20.18$ & 4 \\
\hline NGC 5701 & $\mathrm{~T} 2:$ & 0.0 & 26.1 & $\ldots$ & $\ldots$ & $\ldots$ & $\ldots$ & $<1.1$ & $<19.95$ & \\
\hline NGC 5746 & $\mathrm{~T} 2$ & 3.0 & 29.4 & $\ldots$ & $\ldots$ & $\ldots$ & $\ldots$ & $<1.0$ & $<20.01$ & 4 \\
\hline NGC 5813 & L2: & -5.0 & 28.5 & 150111.228 & 014207.14 & 1.6 & 2.2 & 2.4 & 20.33 & 5 \\
\hline NGC 5838 & $\mathrm{~T} 2::$ & -3.0 & 28.5 & $a$ & $a$ & $\ldots$ & 1.6 & 1.6 & 20.19 & 3 \\
\hline NGC 5846 & $\mathrm{~T} 2:$ & -5.0 & 28.5 & $a$ & $a$ & $\ldots$ & 6.3 & 6.3 & 20.79 & 3 \\
\hline NGC 5850 & L2 & 3.0 & 28.5 & $\ldots$ & $\ldots$ & $\ldots$ & $\ldots$ & $\ldots$ & $\ldots$ & 1 \\
\hline NGC 5866 & $\mathrm{~T} 2$ & -1.0 & 15.3 & 150629.500 & 554547.58 & 0.4 & 7.1 & 7.5 & 20.30 & \\
\hline NGC 5879 & $\mathrm{~T} 2 / \mathrm{L} 2$ & 4.0 & 16.8 & $\ldots$ & $\ldots$ & $\ldots$ & $\ldots$ & $<1.1$ & $<19.57$ & \\
\hline NGC 5921 & $\mathrm{~T} 2$ & 4.0 & 25.2 & $\ldots$ & $\ldots$ & $\ldots$ & $\ldots$ & $<1.0$ & $<19.88$ & 4 \\
\hline NGC 5970 & $\mathrm{~L} 2 / \mathrm{T} 2:$ & 5.0 & 31.6 & $\ldots$ & .. & $\ldots$ & $\ldots$ & $\ldots$ & $\ldots$ & 1 \\
\hline NGC 5982 & L2:: & -5.0 & 38.7 & $\ldots$ & .. & $\ldots$ & $\ldots$ & $\ldots$ & $\ldots$ & 1 \\
\hline NGC 5985 & L2 & 3.0 & 39.2 & $\ldots$ & .. & $\ldots$ & $\ldots$ & $\ldots$ & & 1 \\
\hline NGC 6340 & $\mathrm{~L} 2$ & 0.0 & 22.0 & $\ldots$ & $\ldots$ & $\ldots$ & $\ldots$ & $<1.5$ & $<19.94$ & \\
\hline NGC 6384 & $\mathrm{~T} 2$ & 4.0 & 26.6 & $\ldots$ & $\ldots$ & $\ldots$ & $\ldots$ & $<1.0$ & $<19.93$ & 4 \\
\hline NGC 6482 & $\mathrm{~T} 2 / \mathrm{S} 2::$ & -5.0 & 52.3 & $\ldots$ & $\ldots$ & $\ldots$ & $\ldots$ & $<1.0$ & $<20.52$ & 4 \\
\hline NGC 6500 & L2 & 1.7 & 39.7 & 175559.782 & 182017.65 & 1.7 & 83.5 & 85.0 & 22.20 & \\
\hline NGC 6503 & $\mathrm{~T} 2 / \mathrm{S} 2:$ & 6.0 & 6.1 & $\ldots$ & $\ldots$ & $\ldots$ & $\ldots$ & $<1.0$ & $<18.65$ & \\
\hline NGC 6703 & L2:: & -2.5 & 35.9 & $\ldots$ & $\ldots$ & $\ldots$ & $\ldots$ & $<1.5$ & $<20.36$ & \\
\hline NGC 6951 & S2 & 4.0 & 24.1 & $\ldots$ & $\ldots$ & $\ldots$ & $\ldots$ & $<1.5$ & $<20.02$ & \\
\hline NGC 7177 & $\mathrm{~T} 2$ & 3.0 & 18.2 & $\ldots$ & $\ldots$ & $\ldots$ & $\ldots$ & $<1.1$ & $<19.64$ & \\
\hline NGC 7217 & L2 & 2.0 & 16.0 & 220752.395 & 312133.65 & 2.9 & $\ldots$ & $<0.6$ & $<19.23$ & 2 \\
\hline NGC 7331 & $\mathrm{~T} 2$ & 3.0 & 14.3 & $\ldots$ & $\ldots$ & $\ldots$ & $\ldots$ & $<1.1$ & $<19.43$ & \\
\hline NGC 7479 & S1.9 & 5.0 & 32.4 & 230456.632 & 121922.65 & 1.0 & 2.4 & 2.5 & 20.48 & \\
\hline NGC 7626 & $\mathrm{~L} 2::$ & -5.0 & 45.6 & 232042.538 & 081300.98 & 2.9 & 39.8 & 40.0 & 22.00 & \\
\hline NGC 7742 & $\mathrm{~T} 2 / \mathrm{L} 2$ & 3.0 & 22.2 & $\ldots$ & $\ldots$ & $\ldots$ & $\ldots$ & $<1.1$ & $<19.81$ & \\
\hline
\end{tabular}


Table 1. continued.

\begin{tabular}{ccccccccccc}
\hline \hline Name & $\begin{array}{c}\text { Activity } \\
\text { Type }\end{array}$ & T & $\begin{array}{c}\text { Dist. } \\
(\mathrm{Mpc})\end{array}$ & $\begin{array}{c}\text { R.A. } \\
(\mathrm{J} 2000)\end{array}$ & $\begin{array}{c}\text { Dec. } \\
(\mathrm{J} 2000)\end{array}$ & $\begin{array}{c}\Delta \\
\left({ }^{\prime \prime}\right)\end{array}$ & $\begin{array}{c}\mathrm{S}_{\text {peak }} \\
(\mathrm{mJy} / \\
\text { beam })\end{array}$ & $\begin{array}{c}\mathrm{S}_{\mathrm{tot}} \\
(\mathrm{mJy})\end{array}$ & $\begin{array}{c}\text { Log L Leak } \\
\left(\mathrm{W} \mathrm{Hz}^{-1}\right)\end{array}$ & $\begin{array}{c}\text { Com. } \\
(8)\end{array}$ \\
\hline (1) & $(2)$ & $(3)$ & $(4)$ & $(5)$ & $(6)$ & $(7)$ & $(9)$ & $(10)$ & $(11)$ \\
\hline NGC 7743 & S2 & -1.0 & 24.4 & 234421.364 & 095603.84 & 1.1 & 1.0 & 1.5 & 19.85 \\
NGC 7814 & L2:: & 2.0 & 15.1 & $\cdots$ & $\cdots$ & $\cdots$ & $\cdots$ & $<1.1$ & $<19.48$ \\
\hline
\end{tabular}

Note. - This table lists all AGNs and low-luminosity AGNs - i.e. all Seyferts, LINERs, and transition nuclei in the Palomar spectroscopic sample of nearby bright galaxies. All appropriate columns are listed for radio data from Nagar et al. 2000, Nagar et al. 2002, and this paper. For sources taken from the literature, not all columns are listed; the listed and the remaining data can be accessed from the reference listed in col 11.

Note. - Columns are: (1) galaxy name; (2) nuclear activity type as given by H97a. 'L' represents LINER, 'S' represents Seyfert, 'H' represents an H II region type spectrum, and 'T' represents objects with transitional 'L' + 'H' spectra. '2' implies that no broad $\mathrm{H} \alpha$ is detected, '1.9' implies that broad $\mathrm{H} \alpha$ is present, but not broad $\mathrm{H} \beta$, and '1.0' or '1.5' implies that both broad $\mathrm{H} \alpha$ and broad $\mathrm{H} \beta$ are detected, with the specific type depending on the ratio of the flux in [O III] $\lambda 5007$ to the flux in broad + narrow H $\beta$ (e.g. Osterbrock 1981). The ':' and '::' symbols represent uncertain, and highly uncertain, classifications, respectively; (3) Hubble morphological parameter T as listed in H97a; (4) distance in Mpc to galaxy, as listed in H97a; (5) and (6) $15 \mathrm{GHz}$ radio position (J2000), from our $15 \mathrm{GHz}$ A-configuration VLA maps, unless otherwise mentioned. Positions in Nagar et al. (2000,2002) were reported in equinox B1950. Here we have precessed the B1950 positions to J2000 using the SCHED software (see text). For entries marked ' $a$ ' the position can be found in the reference listed in column 11; (7) offset, in arcsec, between the $15 \mathrm{GHz}$ radio position and the position of the galaxy optical nucleus listed in Cotton et al. 1999; (8) and (9) peak and total flux-density, obtained by fitting a single Gaussian (with peak flux-density, major and minor axes as free parameters) to the radio nuclear source in our maps; (10) logarithm of the radio luminosity (derived from the peak radio flux); (11) Comments as listed below.

Note. - Comments: 1. these are the only 4 LLAGNs in the sample which remain unobserved at high resolution; 2. radio position from VLA D-configuration maps (Nagar et al. 2000); 3. $15 \mathrm{GHz}$ flux estimated from data in Filho et al. (2004); 4. not detected in $\sim 1^{\prime \prime} 5$ resolution $8.4 \mathrm{GHz}$ VLA maps (Filho et al. 2002, 2004) and thus taken to be $<1 \mathrm{mJy}$ at $15 \mathrm{GHz} ; \mathbf{5}$. $15 \mathrm{GHz}$ flux and position from Birkinshaw et al. (priv. communication); derived from 0!' 4 resolution $5 \mathrm{GHz}$ VLA imaging; 6. this is a tentative $(<5 \sigma)$ detection; 7. $15 \mathrm{GHz}$ flux from Carral et al. (1990); 8. $15 \mathrm{GHz}$ flux estimated from data in Ulvestad \& Wilson (1989); 9. $15 \mathrm{GHz}$ flux estimated from data in Venturi et al.(1993); 10. $15 \mathrm{GHz}$ flux estimated from data in Dhawan et al. (1998); 11. $15 \mathrm{GHz}$ flux estimated from data in Jones \& Wehrle (1997); 12. $15 \mathrm{GHz}$ flux estimated from data in Wrobel \& Heeschen (1984).

above a flux limit of 1-1.5 mJy. Additionally, one H II type nuclei (NGC 3690) was also detected. Alternatively, one can state that radio nuclei with luminosity $L_{15}^{\text {core }} \mathrm{GHz} \geq 10^{20} \mathrm{~W} \mathrm{~Hz}^{-1}$ are found in 15 of 45 Seyferts, 27 of 84 LINERs, and 6 of 64 transition nuclei. The radio luminosities of the detected $15 \mathrm{GHz}$ nuclei lie between $10^{18}$ and $10^{23} \mathrm{~W} \mathrm{~Hz}^{-1}$, similar to the luminosities seen in "normal" E/S0 galaxies (Sadler et al. 1989). It is notable, however, that a significant fraction of the detected $15 \mathrm{GHz}$ compact nuclei are in spiral galaxies. Most of the detected $15 \mathrm{GHz}$ nuclear radio sources are compact at the 0 .' 15 resolution (typically $15-25 \mathrm{pc}$ ) of our survey: the implied brightness temperatures are typically $T_{\mathrm{b}} \geq 10^{2.5-4.0} \mathrm{~K}$.

A complete list of results of the VLA observations of all Palomar LLAGNs and AGNs appears in Table 1 with columns explained in the footnotes. All table columns are listed for sources observed by us; for data taken from the literature we list only the peak and total flux, the peak luminosity and the reference in which these and the remaining data of the table can be found. For the new VLA observations reported here (Sect. 3.1) the radio positions of detected nuclei were measured directly in J2000 coordinates. For detections reported earlier in Nagar et al. (2000) and Nagar et al. (2002a), we have precessed the B1950 coordinates reported there to equinox J2000
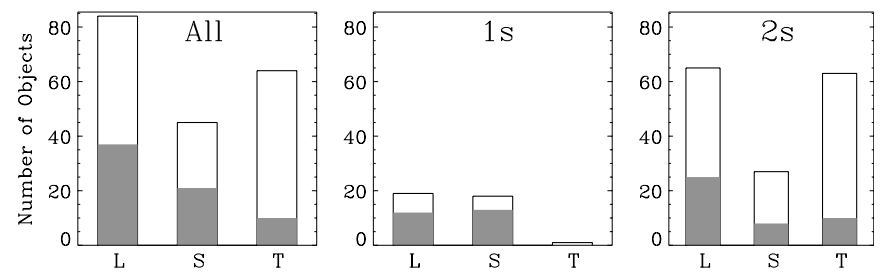

Fig. 1. Detection rate of $15 \mathrm{GHz} 150$-mas-scale radio nuclei for "L"INERs, "S"eyferts, and "T"ransition nuclei in the Palomar sample. The total number of objects is shown by the upper histogram and the number detected is shown by the grey-shaded histogram. Note the higher detection rates of type 1 (i.e. galaxies with broad $\mathrm{H} \alpha$ emission) Seyfert and LINER nuclei.

using the SCHED software (the software used to create VLBA observing scripts). The radio positions for the detected nuclei are limited by the positional accuracy of the phase calibrators (typically 2-10 mas), and by the accuracy of the Gaussian fit to the source brightness distribution, which depends on the signalto-noise ratio of the source detection. The overall accuracy should typically be better than $\sim 50$ mas. We have compared the radio positions derived here with optical positions from 


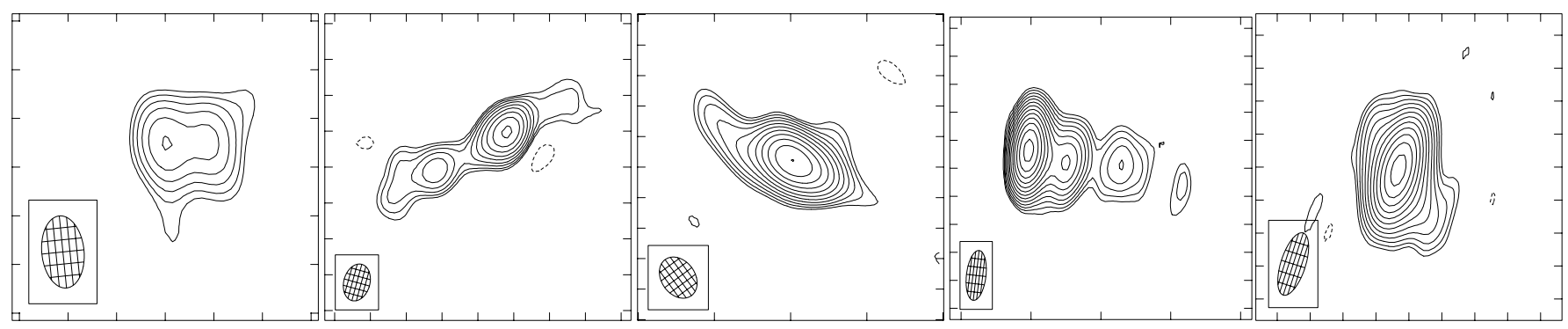

Fig. 2. $5 \mathrm{GHz}(6 \mathrm{~cm})$ VLBA maps of (left to right) NGC 2273, NGC 4589, NGC 5353, NGC 5363, and NGC 7626. The contours are integer powers of $\sqrt{2}$, multiplied by the $2 \sigma$ noise level of $0.25 \mathrm{mJy}(0.3 \mathrm{mJy}$ in the case of NGC 5353). The peak flux-densities are 20.6, 6.1, 13.7, 26.1, and $19.0 \mathrm{mJy} /$ beam, respectively. Tick marks are every 2 mas except for NGC 5353 and NGC 5363 (5 mas).

Cotton et al. (1999a), which were measured from the digital sky survey with typical $1 \sigma$ accuracy $11^{\prime \prime} 5-2$ '. 5 in each of right ascension and declination. The results (Col. 7 of Table 1) show a good $(\leq 2 \sigma)$ agreement in most cases. Very few nuclei show reliable extended structure in our $15 \mathrm{GHz}$ maps; the absence of extended emission in most nuclei is not surprising as the high resolution may resolve it out. In addition, such extended emission is expected to be weak at the high frequency observed.

For all the 8 additional sources (i.e. not in the Palomar sample) with accurate black hole masses and observed in the January 2001 VLA run (Sect. 3.1), we can place firm $5 \sigma$ upper limits of $1.5 \mathrm{mJy}$ on the nuclear radio emission.

\subsection{Results of the VLBA or VLBI observations}

All ten LLAGNs newly observed with the VLBA at $5 \mathrm{GHz}$ were clearly detected in initial maps (i.e. without any form of self-calibration). The single H II type nucleus observed, NGC 3690, was not detected in our observations; a weak $(1.5 \mathrm{mJy})$ high brightness-temperature $\left(>10^{7} \mathrm{~K}\right)$ radio nucleus in this source was detected in previous deep $1.4 \mathrm{GHz}$ VLBI observations (Lonsdale et al. 1993). Of the nuclei detected in our observations, mas-scale radio cores were already known to exist in NGC 1167 (Sanghera et al. 1995; Giovannini et al. 2001), NGC 2911 (Schilizzi et al. 1983; Slee et al. 1994; Filho et al. 2002), NGC 5353, NGC 5363 (Hummel et al. 1982), and NGC 7626 (Xu et al. 2000). Additionally, NGC 2273 (Lonsdale et al. 1992) was also suspected of having a mas-scale radio core. Contour maps of the sources found to be extended in our new VLBA observations are shown in Fig. 2. Images of the other radio nuclei with extended mas-scale structure can be found in Falcke et al. (2000), Nagar et al. (2002a, and references therein), Anderson et al. (2004), and Filho et al. (2004).

The VLBA observations confirm that all except one (NGC 2655) nucleus with $S_{\mathrm{VLA}}^{15 \mathrm{GHz}}>2.7 \mathrm{mJy}$ are genuine AGNs with the radio emission coming from mas- or sub-parsecscales. A compilation of the results of all VLBA and VLBI observations of LLAGNs and AGNs in the Palomar sample is presented in Table 2, with columns explained in the footnotes. Of the 44 sources listed in the table, 39 are from the flux-limited sample (i.e. $S_{15 \mathrm{GHz}}^{\mathrm{VLA}}>2.7 \mathrm{mJy}$ in Table 1). Radio positions of sources with data taken from the literature can be found in the references listed in Col. 18. For sources from this and our previous works we list source positions referenced to the positions of their respective phase-calibrators. All of these source positions have been updated to reflect the latest (as of January 2004) phase calibrator positions (expected accuracy $\sim 1$ mas; Beasley et al. 2002). The other factors contributing to the position uncertainty are the accuracy of the Gaussian fit to the target source - which should typically be better than 2 mas and the error in transfering the phase-calibrator position to the source - which is expected to be better than 3 mas given the small angular separations between our source-phase-calibrator pairs (see e.g. Fig. 3 of Chatterjee et al. 2004). Thus the overall accuracy of the positions listed in Table 2 should be better than 5 mas. The implied brightness temperatures were calculated using the formula given in Falcke et al. (2000); the results are in the range $>10^{6.3} \mathrm{~K}$ to $\gtrsim 10^{10.8} \mathrm{~K}$. Since most of the sources are unresolved, these values are lower limits to the true brightness temperatures.

It is interesting to note that the VLBI-detected nuclei in Table 2 include two galaxy pairs in which both members of the pair host an AGN. NGC 3226 and NGC 3227 (which together form Arp 94) are a galaxy pair (inter-nuclear distance $\sim 2$.2 or $\sim 13 \mathrm{kpc}$ ) in a strong encounter with prominent tidal plumes. NGC 5353 and NGC 5354 have a nuclear separation of $\sim 1$.2 or $\sim 12 \mathrm{kpc}$, and are members of Hickson compact group 68 (HCG 68).

\section{Radio properties}

\subsection{Correlations between radio luminosity, optical emission-line and host galaxy properties}

Correlations between radio, optical emission line, and host galaxy properties in the Palomar Sample have been addressed in detail for all Seyferts (Ulvestad \& Ho 2001a), all (96) LLAGNs at $D \leq 19 \mathrm{Mpc}$ (Nagar et al. 2002a), and all transition nuclei (Filho et al. 2004). The results from these papers are essentially unchanged after expanding the sample to include all LLAGNs and AGNs in the Palomar sample. Here we present only an update on the relation between radio luminosity and optical emission-line luminosity (Fig. 3). The elliptical radio detections (filled symbols in the left panel) are closely related to FR Is, as found earlier by Nagar et al. (2002a). The late-type Palomar Seyferts detected in the radio (filled triangles in the right panel) on the other hand lie closer to the region (and its low luminosity extrapolation) occupied by "classical" Seyferts (see also Nagar et al. 2002a). 


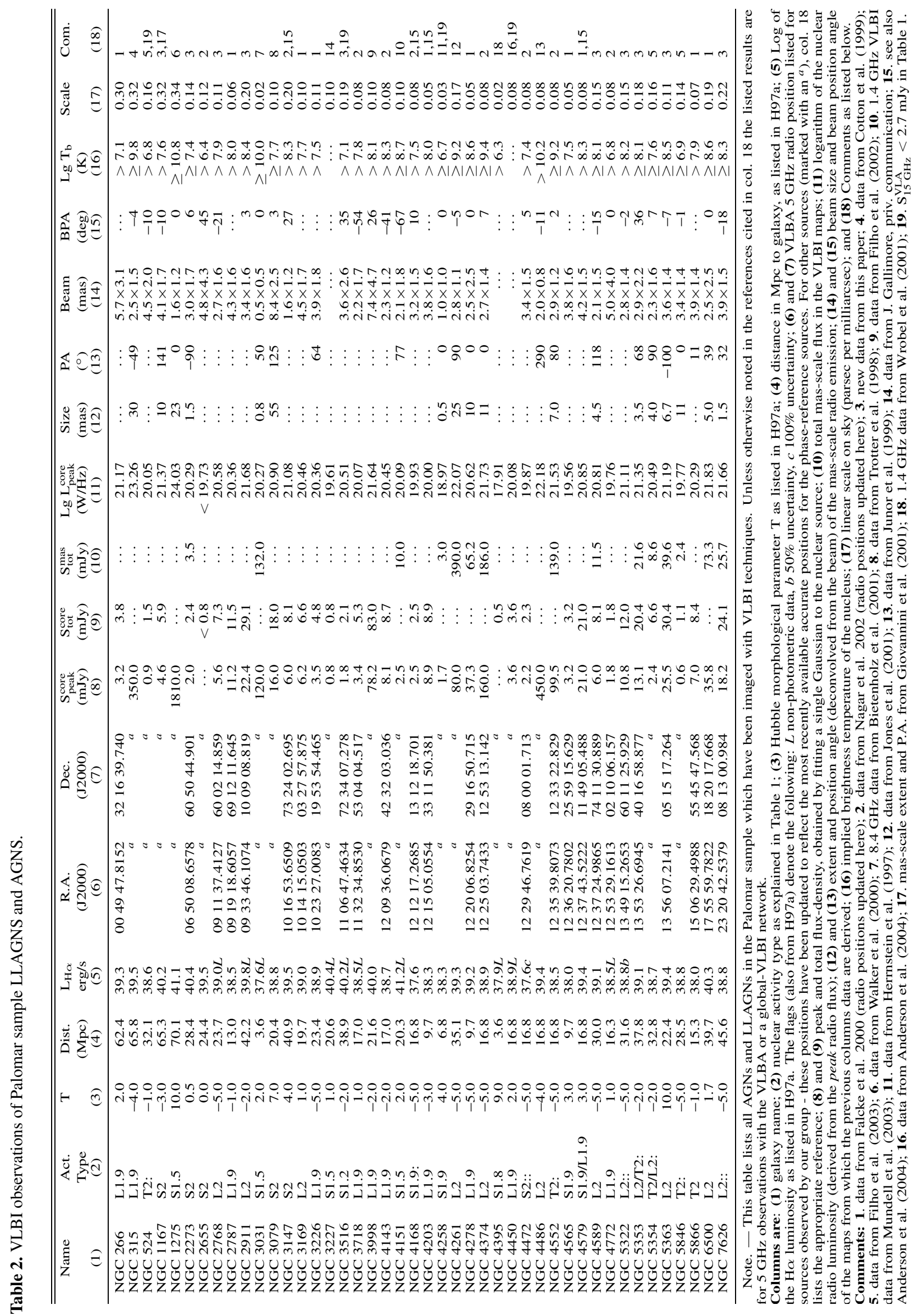



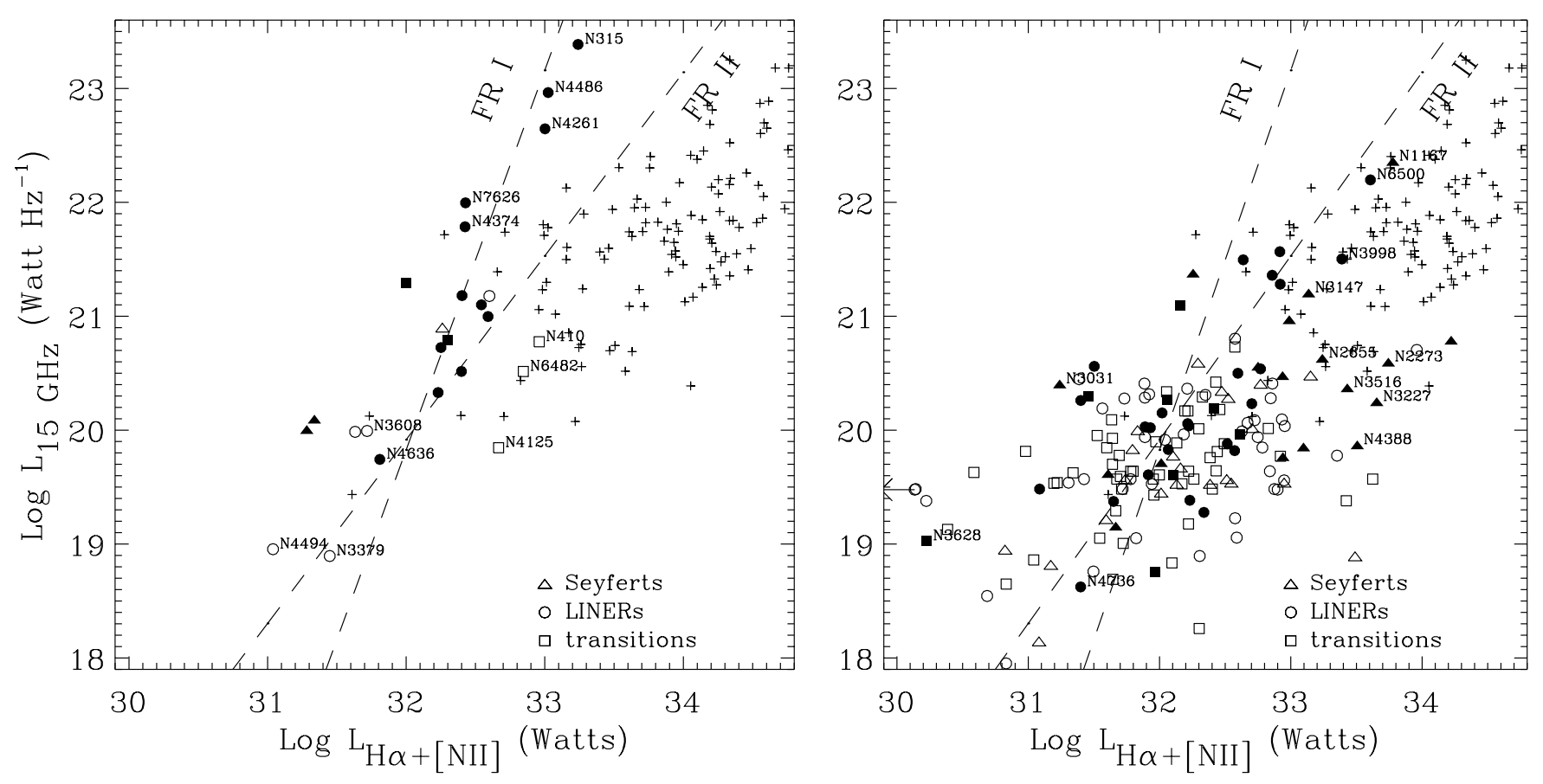

Fig. 3. A plot of the log of the $15 \mathrm{GHz}$ nuclear (150 mas resolution) radio luminosity versus nuclear $\mathrm{H} \alpha+[\mathrm{N}$ II] $\lambda \lambda 6548,6583$ luminosity for all LLAGNs and AGNs in the Palomar sample in elliptical (left) and non-elliptical (right) host galaxies. In both panels, Seyferts, LINERs, and transition nuclei from the Palomar sample are shown as triangles, circles, and squares, respectively. For these, filled symbols are used for radio detected nuclei and open symbols are used for upper limits to the radio luminosity. For comparison, radio-detected "classical" Seyfert galaxies (from Whittle 1992a, i.e. not from the Palomar Sample) are plotted as crosses in both panels, regardless of their galaxy morphological type (for these the [O III] luminosity was converted to an $\mathrm{H} \alpha+[\mathrm{N} \mathrm{II}]$ luminosity using standard flux ratios for Seyferts; see Nagar et al. 2002a). Also shown are the low-luminosity extrapolations of linear fits to the same relationship for FR I and FR II radio galaxies (dashed lines; Zirbel \& Baum 1995).

\subsection{Radio luminosity and black hole mass}

Correlations between radio luminosity, black hole mass, and galaxy luminosity have been discussed in Nagar et al. (2002a) using all of the 150 mas resolution VLA radio data listed in this paper along with sub-arcsecond resolution radio data on other nearby galaxies with black hole mass $\left(M_{\mathrm{BH}}\right)$ estimates. Nagar et al. (2002a) found that the radio luminosity is correlated with both the black hole mass and the bulge luminosity at the $99.99 \%$ significance level. Partial correlation analysis on the two correlations yielded the result that each correlation is meaningful even after removing the effect of the other correlation. Since then a "fundamental plane" between black hole mass, X-ray luminosity, and radio luminosity, which fits both Galactic black hole candidates and AGNs has been claimed by Merloni et al. (2003) and Falcke et al. (2004).

Here we refine the correlations presented in Nagar et al. (2002a) by considering only nuclei observed with linear resolution $\leq 1 \mathrm{pc}$ in the radio and for which one radio component can be relatively unambiguously identified with the location of the central engine. This resolution and morphological criterion enables a more accurate measure of the radio emission from only the accretion inflow and/or the sub-parsec base of the jet, and helps avoid contamination from radio emission originating in knots further out in the jet. The latter radio emission is common in LLAGNs (Sect. 5.4) and often dominates the parsec scale radio emission in Seyferts. In fact many Seyferts have several radio sources in the inner parsec, none of which are unambiguously identifiable with the central engine (Kukula et al. 1999; Mundell et al. 2003; Middelberg et al. 2004).

Figure 4 shows the relation between sub-parsec radio luminosity, $M_{\mathrm{BH}}$, and host galaxy bulge luminosity in the $B$-band. Black hole masses are either directly measured from stellar, gas, or maser dynamics, or estimated from the central stellar velocity dispersion, $\sigma_{\mathrm{c}}$ (Emsellem et al. 1999; Gebhardt et al. 2003, for all other references see Nagar et al. 2002a). We used the relationship of Tremaine et al. (2002) to estimate $M_{\mathrm{BH}}$ from $\sigma_{\mathrm{c}}$. For the circles and triangles in the plot, we also show the error in the black hole mass determination (Fig. 4a). These errors represent reported $1 \sigma$ errors for masses measured directly from stellar, gas, or maser dynamics (e.g. Gebhardt et al. 2003), or reflect $1 \sigma$ errors in the reported central stellar velocity dispersion $\left(\sigma_{\mathrm{c}}\right)$ assuming no additional error in converting $\sigma_{\mathrm{c}}$ to black hole mass (Tremaine et al. 2002).

The plotted circles show the 44 galaxies in Table 2, except NGC 266 (linear resolution $1.3 \mathrm{pc}$; plotted as a cross), NGC 1167, NGC 4772 (no measurement of $\sigma_{\mathrm{c}}$ in the literature), and NGC 2655 (not detected with the VLBA). NGC 4395 (in the Palomar sample, and detected in deep VLBA observations; Wrobel et al. 2001) is taken to not have a bulge (Filippenko \& Ho 2003). In addition, we plot (as triangles) 6 galaxies which are not Palomar LLAGNs or Palomar AGNs and which have radio nuclei relatively unambiguously identified with the central engine in maps with resolution better than $1 \mathrm{pc}$, and available black hole mass measurements or estimates from $\sigma_{\mathrm{c}}$. These 6 galaxies are: the Galaxy 


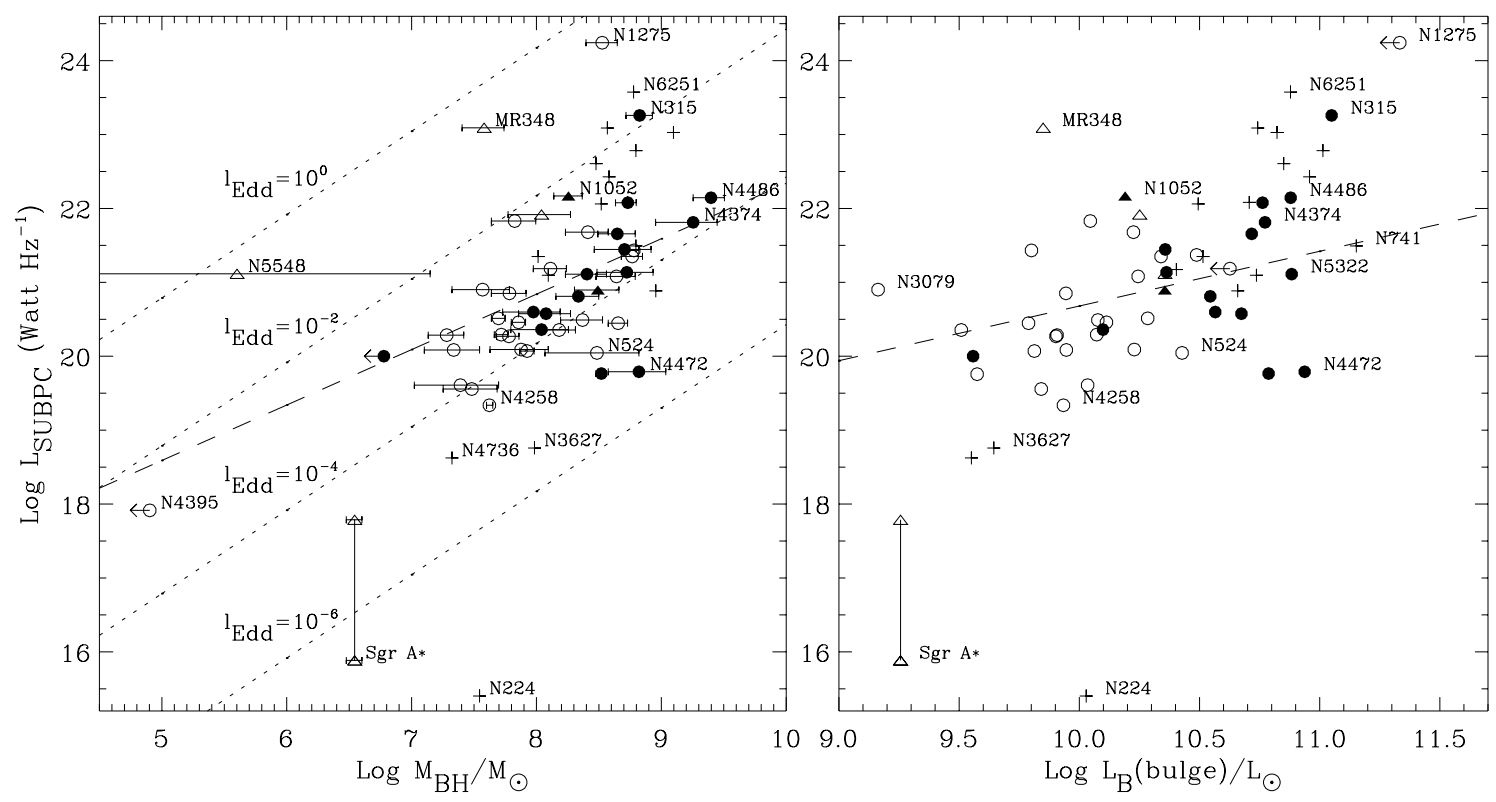

Fig. 4. A plot of sub-parsec radio luminosity versus (left) black hole mass and (right) bulge luminosity of the host galaxy in the $B$-band. Only radio-detected sources relatively unambiguously identified with the central engine of the AGN (see text) and with radio fluxes measured at resolution better than $1 \mathrm{pc}$ are plotted as circles (Palomar LLAGNs and AGNs) and triangles (other LLAGNs and AGNs). For these, filled symbols are used for elliptical galaxies, and errors in black hole mass are shown (see text). LLAGNs and AGNs (some of which are in the Palomar sample) with radio luminosities measured at resolution between $1 \mathrm{pc}$ and $5 \mathrm{pc}$ are shown as crosses. Two measurements (at different resolutions; Nagar et al. 2002a) are plotted for the Galaxy. The four dotted lines in the left panel represent Eddington ratios (from $10^{-6}$ to 1 ) calculated assuming that jet kinetic power dominates the accretion energy output (see Sect. 5.5). The dashed line in the left panel shows a linear fit to the circles and triangles with $\log M_{\mathrm{BH}}>10^{7} M_{\odot}$; the dashed line in right panel shows a linear fit to the circles and triangles except the Galaxy (see text).

(Krichbaum et al. 1998), for which as in Nagar et al. (2002a) we plot two radio luminosities: that of only Sgr A* (10 mas or $4 \times 10^{-4} \mathrm{pc}$ resolution) and that for the full Sgr complex; and Seyferts observed with the VLBA/I (from the list compiled in Middelberg et al. 2004): Mrk 348 (Ulvestad et al. 1999), NGC 1052 (Kellermann et al. 1998), NGC 2110, NGC 5252 (Mundell et al. 2000), and NGC 5548 (Wrobel 2000).

Xu et al. (2000) observed additional (i.e. not in the Palomar Sample) FR Is with the VLBA, but their resolution of $\sim 7$ mas translates to $>1 \mathrm{pc}$ and only some of these FR Is could be included in the figure as crosses. We do not consider other more powerful radio sources (e.g. blazars) to minimize confusion due to relativistic beaming.

A visual inspection of Fig. 4 shows a rough overall correlation between radio luminosity and both black hole mass and galaxy bulge luminosity. The large scatter in both relationships is possibly due to a large range of accretion rates at any given black hole mass (dotted lines in Fig. 4 left), which results in widely different output radio luminosities. Statistical tests $^{3}$ from the ASURV package (Lavalley et al. 1992), suggest that both correlations are statistically significant even when the Galaxy and other nuclei with low black hole mass are removed, as detailed below. The radio luminosity and black hole mass correlation has significance $99.95-99.98 \%$ when all circles and triangles in Fig. 4 (left panel) are considered. This significance drops only slightly (98.9-99.8\%) when the Galaxy

\footnotetext{
${ }^{3}$ Measurement errors were not considered when running the statistical tests.
}

and NGC 4395 are not considered, and is still $98.9-99.8 \%$ when only nuclei with $\log M_{\mathrm{BH}}>10^{7} M_{\odot}$ are considered. The correlation between radio luminosity and bulge luminosity has significance $99.3-99.7 \%$ when all circles and triangles in Fig. 4 (right panel) are considered. This correlation is still significant (95.6-99.2\%) when the Galaxy is not considered. Linear regression analysis by the Buckley James method in ASURV on the circles and triangles in Fig. 4 (not considering the Galaxy in both cases, and using only nuclei with $\log M_{\mathrm{BH}}>10^{7} M_{\odot}$ in the case of the relation between radio luminosity and $M_{\mathrm{BH}}$ ) yields:

$$
\begin{aligned}
& \log \left(L_{\text {Sub-pc }}[\mathrm{W} / \mathrm{Hz}]\right) \\
& \quad=0.74( \pm 0.31) \log \left(L_{\mathrm{B}}(\text { bulge }) / L_{\odot}\right)+13.28 \\
& \quad=0.75( \pm 0.26) \log \left(M_{\mathrm{BH}} / M_{\odot}\right)+14.84 .
\end{aligned}
$$

These two relations are plotted with dashed lines in Fig. 4.

The relation between radio luminosity and black hole mass has been discussed by several authors, with conflicting results. For example, Franceschini et al. (1998) claimed a correlation with a much steeper slope based on a small number of objects, while Ho (2002) and Woo \& Urry (2002) found no correlation for larger samples of AGNs and LLAGNs. We emphasize that our results and those of Ho (2002) and Woo \& Urry (2002) are not contradictory given the samples and physical scales of the radio emission. Here, in Fig. 4, we specifically address the correlation between radio emission from the base of the jet or from the innermost accretion inflow (measured here by the subparsec radio emission in nuclei for which this radio emission is 


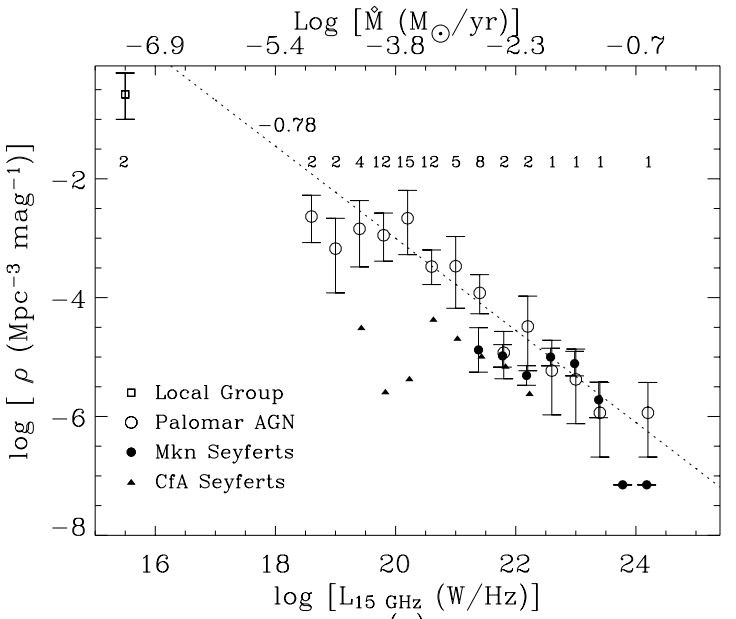

(a)

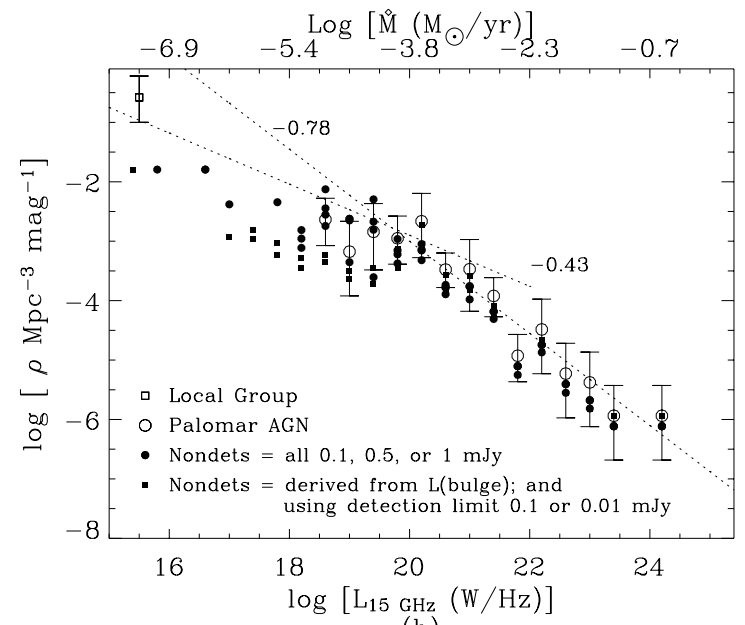

(b)

Fig. 5. a) The $15 \mathrm{GHz}$ radio luminosity function (RLF) of the 150 mas-scale radio nuclei in the LLAGNs and AGNs of the Palomar sample (open circles, with the number of galaxies in each bin listed above the symbol). For a rough comparison (see text) we also plot the $15 \mathrm{GHz}$ RLFs (after converting to our value of $H_{0}$ and frequency; see text) of Markarian Seyferts and CfA Seyferts. The dotted line is a power-law (-0.78) fit to the Palomar nuclear RLF (excluding the two lowest radio luminosity points). Also shown is the estimated $15 \mathrm{GHz}$ nuclear RLF of galaxies in the local group (open square, with 2 galaxies; see text). The upper $x$ axes of both panels show the implied logarithm of the mass accretion rate (in $M_{\odot} \mathrm{yr}^{-1}$ ) within the context of a jet model (Falcke \& Biermann 1999), assuming both a $10 \%$ energy conversion efficiency, and that the jet kinetic power dominates the accretion energy output (see Sect. 5.5); b) the axes and open symbols are the same as in a). The filled symbols show several simulated nuclear RLFs for the Palomar sample which include nuclei not detected in our $15 \mathrm{GHz}$ survey in the RLF calculation (see text for details). The dotted line with slope -0.43 shows a possible fit (made by eye) to the RLF at the lowest luminosities; the other dotted line is the same as in the left panel.

relatively unambiguously associated with the true "core" rather than with larger scale "jets") and black hole mass or bulge luminosity for a sample dominated by nearby LLAGNs. It is possible that the range of actual Eddington ratios $l_{\text {Edd }}$ (which could be responsible for the scatter in radio luminosities at any given black hole mass) is small enough among LLAGNs in our sample to not destroy the correlation between sub-parsec radio luminosity and black hole mass or bulge luminosity. Indeed, the estimated $l_{\text {Edd }}$ for the nuclei in Fig. 4 (calculated assuming the jet power, $Q_{\text {jet }}$, dominates the accretion energy output; see Sect. 5.5) spans a relatively narrow range (see points and dashed lines in the left panel of Fig. 4). Ho (2002), on the other hand, used a sample with a larger range of $l_{\text {Edd }}$ and measured nuclear radio luminosities at lower resolution $\left(5^{\prime \prime}\right.$; or several hundreds of parsecs). Both factors ("classical" Seyferts are apparently more likely to produce radio emission on 100-pc scales than LLAGNs; see Sect. 5.4) could have lead to the absence of a correlation between radio luminosity and black hole mass in his sample. Similar factors would also apply to the results of Woo \& Urry (2002), who used a sample which covered a larger range of $l_{\text {Edd }}$ and which included many powerful (and presumably relativistically beamed) radio sources.

\subsection{Radio luminosity function}

The nuclear (150 mas-scale) $15 \mathrm{GHz}$ RLF for all 68 radiodetected Palomar sample LLAGNs and AGNs (Table 1) is plotted in Fig. 5a as open circles. LLAGNs not detected in the radio have been excluded from the RLF calculation. Only 2 of the radio detections in the Palomar sample (NGC 1275 and NGC 4151) are true AGNs as defined by their emission-line properties i.e. they have $L_{\mathrm{H} \alpha} \gg 10^{40} \mathrm{erg} \mathrm{s}^{-1}$. The H II-region nuclei and absorption-line nuclei, which are excluded from this RLF calculation, have much lower radio luminosities than the LLAGNs and AGNs in the sample. The RLF has been computed via the bivariate optical-radio luminosity function (following the method of Meurs \& Wilson 1984), after correcting for the incompleteness (Sandage et al. 1979) of the RSA cata$\log$ (from which the Palomar sample was drawn). Errors were computed following the method of Condon (1989). We emphasize that the nuclear $15 \mathrm{GHz}$ RLF presented here traces only the inner AGN jet or accretion inflow, and does not include the contribution from $>150$ mas-scale radio jets.

RLFs at $1.4 \mathrm{GHz}$ and $5 \mathrm{GHz}$ for Palomar Seyferts have been presented in Ulvestad \& Ho (2001a), and a RLF (using observations at several frequencies and resolutions) for the complete Palomar sample has also been discussed in Filho (2003, Ph.D. Thesis). The RLF we present here (first presented in Nagar 2003) is in rough agreement with the above RLFs given the errors. The advantages of the RLF presented here are threefold. First, it is based on a larger number (68) of radio detections. Second, it is derived from uniform radio data: all except 13 radio detections and 21 radio non-detections have their fluxes or upper limits derived from our $15 \mathrm{GHz}(2 \mathrm{~cm})$ VLA A-configuration observations reduced in a uniform way; these 34 exceptions have fluxes or upper limits derived from data of similar resolution and frequency. Third, the radio data were obtained at high resolution and high frequency: both these factors reduce the contamination of star-formation-related emission to the true AGN radio emission, which is especially important at these low AGN luminosities.

At the highest luminosities the Palomar RLF is in good agreement with that of "classical" Seyferts (Fig. 5a), as previously noted by Ulvestad \& Ho (2001a). We have plotted 
the RLFs of Markarian Seyferts (1.4 GHz RLF from Meurs \& Wilson 1984) and of CfA Seyferts (1.4 GHz RLF calculated by Ulvestad \& Ho (2001a) using $8 \mathrm{GHz}$ data from Kukula et al. (1995)), after conversion to our values of $H_{0}$ and frequency (assuming that the $1.4 \mathrm{GHz}$ emission is optically-thin with spectral index -0.75). Of course, the "classical" Seyfert RLFs are not strictly comparable to ours since our $15 \mathrm{GHz}$ survey detected flat-spectrum emission (Nagar et al. 2001, 2002b) which may have been invisible to the $1.4 \mathrm{GHz}$ observations, and conversely, the $15 \mathrm{GHz}$ observations may not have detected the steep spectrum emission which dominated the $1.4 \mathrm{GHz}$ observations. Furthermore, the AGN-related radio structures in the Palomar LLAGNs are either sub-arcsec (i.e. the nuclear radio emission is the total AGN-related radio emission) or, in a few cases, FR I-like. Neither of these can be easily compared or corrected to the radio structures seen in most Markarian or CfA Seyferts at lower radio frequencies.

At lower luminosities, the sample extends the RLF of powerful AGNs by more than three orders of magnitude. A linear (in log-log space) fit to the Palomar nuclear RLF above $10^{19}$ Watt $\mathrm{Hz}^{-1}$ (i.e. excluding the two lowest luminosity bins; see below) yields:

$$
\begin{aligned}
\log \rho= & \left(12.5-0.78 \times \log \left(L_{15 \mathrm{GHz}}\left[\mathrm{W} \mathrm{Hz}^{-1}\right]\right)\right) \\
& {\left[\mathrm{Mpc}^{-3} \mathrm{mag}^{-1}\right] . }
\end{aligned}
$$

As we discuss below, a potential fit to the RLF below

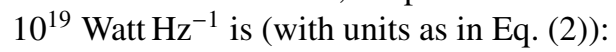

$\log \rho=5.7-0.43 \times \log L_{15 \mathrm{GHz}}$.

There is some indication of a low luminosity turnover in the Palomar RLF (Fig. 5a). Admittedly, this apparent turnover is partly due to the incompleteness of the radio survey, i.e. biased by the sub-milli-Jansky population which remains undetected. Nevertheless there are several reasons to believe the presence of such a turnover, as detailed below and in Fig. 5b. First, and most convincingly, one runs out of bright galaxies: an extension of the -0.78 power law fit to lower luminosities would require e.g. an LLAGN like Sgr A* or M $31 *$ to be present in every $\mathrm{Mpc}^{-3}$. However, there are only about 318 known non-irregular galaxies at $D<10 \mathrm{Mpc}$ of which only 70 have $\log \left(L / L_{\odot}\right)>9$ and only 22 have $\log \left(L / L_{\odot}\right)>10$ (Karachentsev et al. 1999). To better determine the RLF shape at lower luminosities, we have calculated an approximate $\mathrm{RLF}^{4}$ for the nuclei of the local group of galaxies. The local group has 13 galaxies with $M_{\mathrm{v}}<-14.8$, of which 6 are classified as Irregular. The remaining 7 have been surveyed at high resolution with the VLA to detection limits between $30 \mu \mathrm{Jy}$ and $1 \mathrm{mJy}$. Our Galaxy ( $\sim 1 \mathrm{Jy}$ for Sgr A*; Krichbaum et al. 1998) and M 31 (0.033 mJy; Crane et al. 1993) have nuclear radio luminosities $\sim 10^{15}$ Watt $\mathrm{Hz}^{-1}$, and the other five all have upper limits to their nuclear radio flux: M 32 (NGC 221,<0.03 mJy; Ho et al. 2003b), NGC 185

\footnotetext{
${ }^{4}$ Here we use the highest resolution radio flux, instead of a "matched" linear-resolution flux. The latter flux is dominated by extra-nuclear (non-AGN related) emission for these lowest luminosity AGNs and is thus not directly comparable to the radio fluxes of the other more luminous radio sources used in the RLF computation.
}

$(<0.12$ mJy; Ho \& Ulvestad 2001), NGC 147 and NGC 205 (<1 mJy; Heckman et al. 1980), and NGC $598(<0.03 \mathrm{mJy}$ from our reduction of $8.4 \mathrm{GHz}$ A-configuration VLA archive data from project AC342). We calculate the local group RLF using a simple $V / V_{\max }$ test for the Galaxy and M 31, assuming a detection limit of $30 \mu \mathrm{Jy}$. This local group point, plotted as an open square in Figs. 5a and 5b, is also consistent with a lowluminosity break in the overall RLF. The error bar on the local group RLF point is from Poisson statistics; the true error, from the generalization of our (a-posterior) local group properties, is likely to be larger.

As a further test we simulated the shape of the Palomar RLF at low luminosities by converting some or all of the LLAGNs not detected in our $15 \mathrm{GHz}$ survey into radio detections as follows (a total of 6 simulated RLFs; filled symbols in Fig. 5b). We first recomputed the RLF assuming that all 125 radio nondetected LLAGNs had radio nuclei with flux $0.5 \mathrm{mJy}$ and using this value as the assumed detection limit of the survey. We then recomputed the RLF for the three cases that the non-detected Seyferts and LINERs (71 nuclei) all had radio nuclei with flux $1 \mathrm{mJy}, 0.5 \mathrm{mJy}$, or $0.1 \mathrm{mJy}$ (and using the corresponding flux as the assumed detection limit of the survey). The above four simulated RLFs are plotted with filled circles in Fig. 5b. To explore two more possibilities, we set the radio flux of individual non-detected LLAGNs to the values expected from the rough proportionality between bulge luminosity and 150 mas-scale radio luminosity for nearby galaxies (dashed line in Fig. 18, lower panel, of Nagar et al. 2002a). We then recomputed the RLF for two assumed detection limits $(0.1 \mathrm{mJy}$ and $0.01 \mathrm{mJy})$ and ensured that the estimated fluxes fell in the range between $1 \mathrm{mJy}$ (our survey's actual detection limit) and the assumed detection limit (if the estimated flux was lower than the assumed detection limit then the nucleus continued to be treated as a non-detection). The resulting two simulated RLFs, calculated from a total of 98 and 170 radio detections, respectively, are plotted with filled squares in Fig. 5b. All the simulated RLFs support a low luminosity break in the Palomar RLF. The actual shape of the low end of the RLF is uncertain and in Fig. 5b and Eq. (3) we show a potential power law fit which satisfies the current data and extrapolations.

\subsection{Sub-parsec jets}

About 20 of the 44 sources in Table 2 have detected subparsec scale (and/or sometimes larger scale) "jets". These include NGC 315 (Cotton et al. 1999b; Fomalont et al. 2000; Giovannini et al. 2001), NGC 1167 (Giovannini et al. 2001, this work), NGC 1275 (Dhawan et al. 1998; Walker et al. 2000), NGC 2273 (this work), NGC 3031 (M 81; Bietenholz et al. 2000), NGC 3079 (Trotter et al. 1998), NGC 4151 (Mundell et al. 2003; Ulvestad et al. 1998), NGC 4258 (Herrnstein et al. 1997), NGC 4261 (e.g. Jones et al. 2001), NGC 4278 (Jones et al. 1984; Falcke et al. 2000), NGC 4374 (M 84; Wrobel et al. 1996, this work), NGC 4486 (M 87; Junor \& Biretta 1995), NGC 4552 (M 89; Nagar et al. 2002a), NGC 4589 and NGC 5353 (this work), NGC 5354 (Filho et al. 2004), NGC 5363 (this work), NGC 5846 (Filho et al. 2004), 
NGC 6500 (Falcke et al. 2000), and NGC 7626 (this work, also tentatively detected by Xu et al. 2000).

The mas-detected radio nuclei fit into four categories, with Seyferts and LINERs preferentially belonging to one or the other category: (a) powerful radio galaxies or low power radio galaxies - NGC 315, NGC 1275, NGC 4261, NGC 4374, NGC 4486 and NGC 7626 - which have an elliptical host, a LINER nuclear spectrum, and collimated sub-parsec to kpc jets; (b) nuclei which do not have detected parsec-scale jets, but have larger (100 pc- or kpc-scale) jets. These are preferentially Seyfert-like nuclei (6 nuclei) though NGC 5846 (transition nucleus) falls in this class; (c) nuclei with detected sub-parsec jets but weak or no known larger scale jets - preferentially in LINERs or transition nuclei. These nuclei - NGC 4278, NGC 4552, NGC 4589, NGC 5353, NGC 5354, and NGC 6500 - typically show curved or highly bent jets (Nagar et al. 2002a; Filho et al. 2004, this work) and proper motion studies (Nagar et al., in prep.) suggest that these jets are frustrated in the inner few parsecs; (d) the remaining $\sim 23$ nuclei in Table 2 do not show extended mas-scale emission but require more detailed study of their $100 \mathrm{pc}$ to kpc scale radio emission. The disappearance of the larger-scale jet in category (c) above, and the morphology of their parsec-scale jets (highly curved or bent) suggests that the jet does not propagate beyond the inner few parsecs, either due to being uncollimated, or because of interaction with the ambient medium. If this is the case, the energy deposited into the inner few parsecs by the jet (next section) is significant, and could potentially be responsible for lowering the larger scale (i.e. outside the accretion disk) gas inflow and thus ultimately the accretion rate.

\subsection{Jet power versus radiated luminosity}

\subsubsection{Jet power domination in Palomar LLAGNs}

With an estimated black hole mass and an emitted luminosity, one can estimate the Eddington ratio, i.e. $l_{\mathrm{Edd}}=$ $L_{\text {Emitted }} / L_{\text {Eddington }}$. Previous calculations of $l_{\text {Edd }}$ for LLAGNs have considered only the radiated component of $L_{\text {Emitted. Since }}$ LLAGNs lack a "big blue bump", the X-ray emission has been thought to dominate the bolometric luminosity (Ho 1999). With most LLAGNs having hard X-ray luminosities of only $\sim 10^{40} \mathrm{erg} \mathrm{s}^{-1}$ or lower, the accretion is inferred to be highly sub-Eddington (Ho et al. 2001; Terashima \& Wilson 2003; Filho et al. 2004).

If, as justified above, the compact radio nuclei and subparsec jets represent emission from the base of a relativistic jet launched close to the black hole, then the kinetic energy in the jet can be quite high. Equation 20 of Falcke \& Biermann (1999) - assuming an average inclination of $45^{\circ}$ - can be used to obtain "minimum jet powers" $\left(Q_{\text {jet }}\right)$ of $10^{40}-10^{45} \mathrm{erg} \mathrm{s}^{-1}$ (Fig. 6, left panel) from the $15 \mathrm{GHz}$ peak VLA flux of radio detected LLAGNs. For LLAGNs with both hard X-ray and radio luminosity available, this jet power greatly exceeds the radiated X-ray luminosity (Fig. 6, right panel). Since the bolometric luminosity $\left(L_{\mathrm{Bol}}\right)$ in electromagnetic radiation is estimated to be only $\sim 3-15 \times L_{0.5-10 \mathrm{kev}}$ for LLAGNs (Ho 1999), this suggests that the accretion power output is dominated
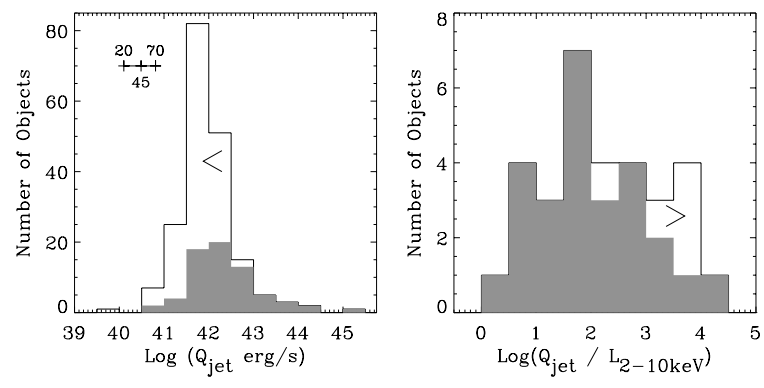

Fig. 6. Left: the implied "minimum jet power", $\left(Q_{\text {jet }}\right)$ of the radiodetected (grey shaded area) and radio non-detected (white area) LLAGNs and AGNs, calculated from the peak VLA $15 \mathrm{GHz}$ flux using Eq. (20) of Falcke \& Biermann (1999) and assuming a jet inclination of $45^{\circ}$ to the line of sight. The inset illustrates the range of calculated minimum jet kinetic powers for three assumed inclinations: $20^{\circ}, 45^{\circ}$, and $70^{\circ}$. Right: $\log$ of the ratio of minimum jet power (assuming $45^{\circ}$ jet inclination) to $\mathrm{X}$-ray luminosity (in the $2-10 \mathrm{keV}$ band) for radio detected LLAGNs and AGNs. The grey and white histograms represent LLAGNs/AGNs with detected, and upper limits to, the hard X-ray emission, respectively.
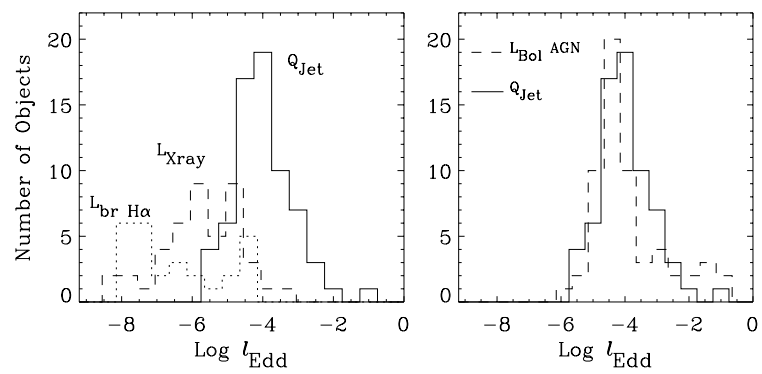

Fig. 7. A comparison of the kinetic and radiated accretion power outputs as a fraction of $L_{\text {Eddington }}$ in four energetically important wavebands. In both panels the histograms are offset by 0.1 in $x$ for clarity. Left: histograms of the broad $\mathrm{H} \alpha$ luminosity (dotted line), hard $\mathrm{X}$-ray luminosity (2-10 keV; dashed line), and minimum jet power ( $Q_{\text {jet }}$; solid line) for all broad line nuclei, all hard X-ray detected nuclei, and all radio detected nuclei, among the LLAGNs and AGNs of the Palomar Sample, respectively. Right: histograms of the minimum jet power $\left(Q_{\text {jet }} ;\right.$ solid line $)$ and radiated bolometric luminosity (derived from the [O III] luminosity, see text; dashed line) for the radio detected nuclei in the LLAGNs and AGNs of the Palomar Sample.

by the jet power. This domination of jet power over X-ray emission is analogous to the situations for $\sim 10-15 M_{\odot}$ black holes in Galactic X-ray binary systems (Fender et al. 2003) and for powerful radio galaxies (e.g. Celotti \& Fabian 1993; Owen et al. 2000).

To expand on this issue, we compare the estimated minimum jet power to the observed hard X-ray luminosity and emission-line luminosities for the Palomar sample LLAGNs. Clearly (Fig. 7, left) the minimum jet power is significantly larger than the measured hard X-ray luminosity (as noted above) and the luminosity in broad $\mathrm{H} \alpha$. On the other hand, if the radiated bolometric luminosity is estimated from the [O III] luminosity - from the empirical result that the spectral energy density of type 1 AGNs typically shows $L_{\mathrm{Bol}}=3500 \times L_{\text {[OIII] }}$ (Heckman et al. 2004, see also next section) - then LLAGNs show similar distributions of minimum jet power and radiated bolometric luminosity (Fig. 7, right). 


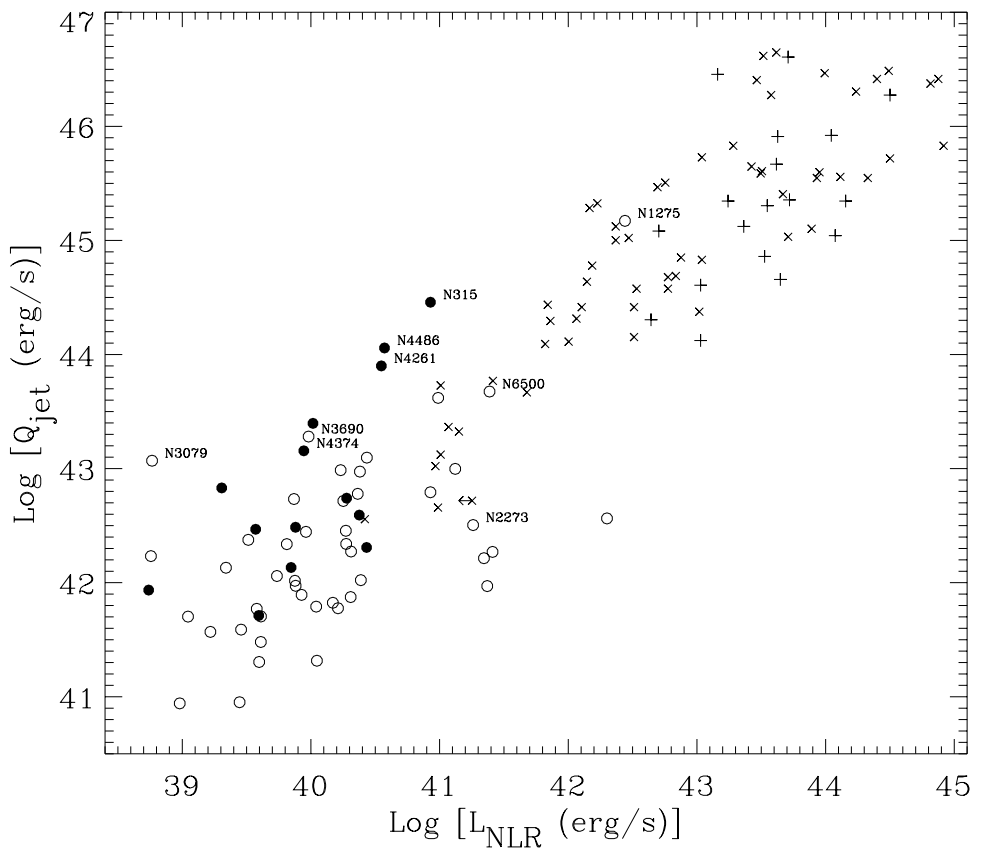

\subsubsection{A common jet-power scaling for LLAGNs and powerful AGNs}

The radio detected LLAGNs in the Palomar sample (circles; Fig. 8) fall nicely at the low luminosity end of the correlation between jet kinetic power and total emission line luminosity from the narrow line region (NLR) for more powerful FR I and FR II galaxies. This figure includes FR I and FR II radio galaxies (slanted crosses; in these the jet kinetic power is estimated from lobe-feeding energy arguments; Rawlings \& Saunders 1991) and other powerful radio sources (including BL Lacs, quasars, and radio galaxies) with parsec-scale jets (crosses; in these the bulk kinetic energy in the jet was estimated using a self-Compton synchrotron model applied to the parsec-scale jet; Celotti \& Fabian 1993). We estimated the NLR luminosity for the Palomar LLAGNs and AGNs following Celotti \& Fabian (1993):

\section{$L_{\mathrm{NLR}}=3 \times\left(3 L_{[\mathrm{OII}] \lambda 3727}+1.5 L_{[\mathrm{OIII}] \lambda 5007}\right)$}

with $L_{[\mathrm{OII}] \lambda 3727}$ estimated as $0.25 \times L_{[\mathrm{OIII}] \lambda 5007}$ for Seyferts and $3 \times L_{[\mathrm{OIII}] \lambda 5007}$ for LINERs and transition nuclei. The Palomar [O III] luminosities were measured in a $2^{\prime \prime} \times 4^{\prime \prime}$ nuclear aperture (Ho et al. 1997a); these values closely approximate the total NLR luminosity in most nuclei except a handful, e.g. NGC 4151, which have more extended NLRs.

\subsubsection{LLAGN jet-power and global energetics}

The energetics of the jet are also important in the context of so called cooling flows and in regulating the feedback between galaxy growth and black hole growth. For example, in many clusters the central $\mathrm{cD}$ galaxy has an FR I radio morphology, with the radio jet playing an important role in the above issues and in the global energetics of the cluster gas (Owen et al. 2000; Binney 2004b; Ostriker \& Ciotti 2004). A comparison of the jet kinetic power with the power injected into the ISM
Fig. 8. A plot of Jet Power (we use the "minimum jet power", $Q_{\text {jet }}$, derived from the nuclear radio luminosity; see text) versus the Narrow Line Region (NLR) luminosity (derived from the [O III] luminosity; see text) for radiodetected nuclei in the Palomar Sample (circles). Filled circles are used for elliptical galaxies in the Palomar sample. These Palomar Sample LLAGNs and AGNs nicely fill in the low luminosity end of similar relationships for FR I and FR II radio galaxies (slanted crosses; Rawlings \& Saunders 1991,with jet kinetic energy estimated from their kpc-scale radio lobes), and other radio galaxies (crosses; Celotti \& Fabian 1993, with jet kinetic energy estimated from their parsec-scale radio jets). The data from other papers have been corrected to the value of $H_{0}$ used in this paper.

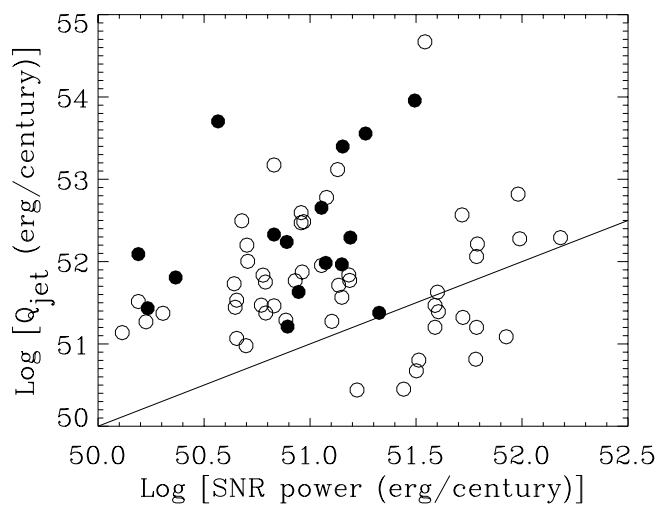

Fig. 9. A comparison of the "minimum jet power" $\left(Q_{\text {jet }}\right)$ and the kinetic energy injected into the ISM by supernovae type I and II for the radio detected Palomar LLAGNs and AGNs. Filled circles are used for elliptical galaxies. The solid line shows the line of equality.

by supernovae types I and II in the host galaxies is shown in Fig. 9 for the radio detected Palomar LLAGNs/AGNs. The supernova rates (as a function of galaxy morphological type) are taken from the "Case B" values in Table 6 of van den Bergh \& McClure (1994), e.g. 0.25 SNu for E and S0 galaxies (all from $\mathrm{SN}$ type Ia), where $1 \mathrm{SNu}=1 \mathrm{SN}$ per century per $10^{10}\left(L_{\mathrm{B}} / L_{\odot}\right)$. The total SN rate is slightly higher in later type galaxies due to the contribution of SN type II. Values of $L_{\mathrm{B}} / L_{\odot}$ were taken from Ho et al. (1997a) and each supernova is assumed to inject $10^{51} \mathrm{erg}$ of kinetic energy into the ISM (e.g. Binney \& Tremaine 1987; Pellegrini \& Ciotti 1998). The jet power is clearly the major player in the nuclear energetics not only because it exceeds the total SN kinetic power in almost all cases, but also because its nuclear origin allows a closer "feedback" to the accretion inflow. A significant fraction of the jet energy is potentially deposited in the central parsecs, especially in LLAGNs which show pc-scale (usually bent) jets but no larger scale jets (Sect. 5.4); such deposition of jet power can considerably slow down or balance any cooling flow or other inflow in 


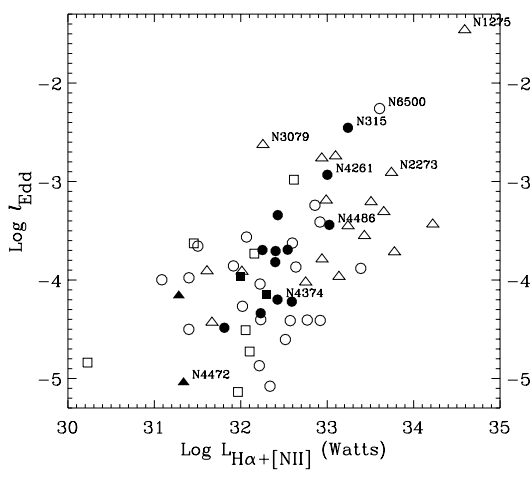

(a)

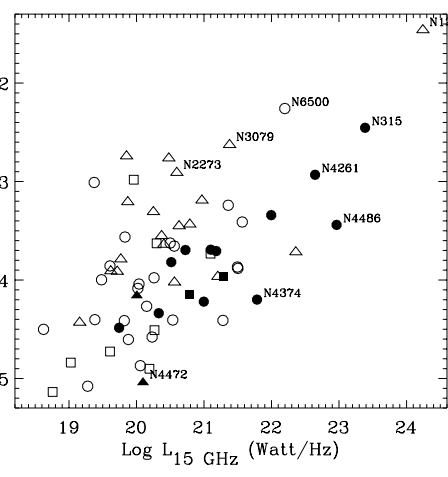

(b)

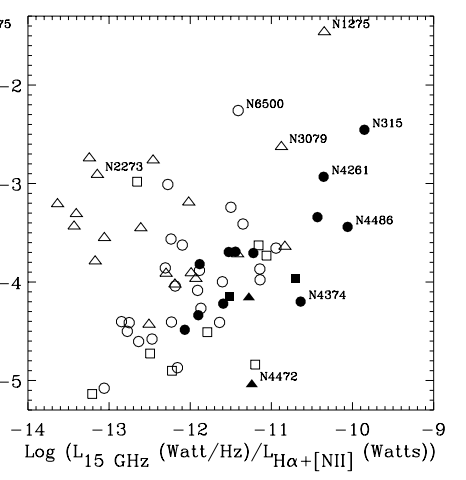

(c)

Fig. 10. Plots of the "minimum jet power" as a fraction of Eddington luminosity (equivalent to the Eddington ratio, $l_{\text {Edd }}$, if the jet power dominates the emitted accretion energy) versus a) the nuclear $\mathrm{H} \alpha+[\mathrm{N} \mathrm{II}] \lambda \lambda 6548,6583$ luminosity; b) the $15 \mathrm{GHz}$ nuclear (150 mas resolution) radio luminosity; and c) ratio of the above two quantities, for all radio-detected LLAGNs and AGNs in the Palomar sample. Seyferts are plotted as triangles, LINERs as circles, and transition nuclei as squares. Filled symbols are used for elliptical galaxies.

the inner parsecs and thus ultimately help "starve" the accretion disk (e.g. Di Matteo et al. 2001). Additionally, LLAGNs with kpc-scale jets inject significant energy into the inter-galactic medium (IGM), and work against any cooling flow (see e.g. Pellegrini \& Ciotti 1998). The most recent of such "feedback" analyses (Binney 2004b) does take into account the jet power, though for the more powerful FR I type jets in cD galaxies. Our results show that their models can be applied, at least qualitatively, to LLAGNs.

In summary, Eddington ratios, $l_{\text {Edd }}$, calculated from hard $\mathrm{X}$-ray luminosities heavily underestimate the true accretion power output in LLAGNs. This finding is in line with that for more powerful radio galaxies, where the jet kinetic power is two to five orders of magnitude larger than the radiated radio luminosity, and often significantly larger than the total radiated bolometric luminosity (Celotti \& Fabian 1993; Owen et al. $2000)$. Using $L_{\mathrm{Bol}}=3500 L_{\text {[OIII }]}$ or $L_{\mathrm{Bol}}=Q_{\text {jet }}$ yields similar distributions of $L_{\mathrm{Bol}}$. This, and the scaling between $L_{\mathrm{NLR}}$ and $Q_{\text {jet }}$, argues for a common central engine in all AGNs from LLAGNs to powerful FR IIs, but with the caveat that the [O III] luminosity in LLAGNs is potentially contaminated by non accretion related processes. Finally, the jet is a significant (maybe even dominant) source of heating in the galaxy. If the jet is disrupted in the inner parsecs, then the jet power could play a role in slowing any cooling flow or other accretion inflow on parsecscales, thus starving the accretion disk.

\subsection{The Eddington ratio}

In the previous section we showed that the accretion energy output in LLAGNs with radio nuclei is dominated by the jet power, and is of the order of $l_{\mathrm{Edd}}=10^{-6}$ to $10^{-2}$. Here we look at the dependence of this jet-power-derived $l_{\text {Edd }}$ on other quantities.

Heckman et al. (2004) have investigated black hole and galaxy growth using 23000 type 2 AGNs from the Sloan Digital Sky Survey (SDSS). They use $\sigma_{\mathrm{c}}$ to estimate black hole mass, and $L_{\mathrm{Bol}}=3500 L_{[\mathrm{OIII}]}$. They find that most present-day accretion occurs onto black holes with masses $<3 \times 10^{7} M_{\odot}$, and that most black hole growth takes place in systems with accretion rate less than one fifth of the Eddington rate. It is interesting to apply their analysis to the Palomar LLAGNs since these objects have emission-line luminosities typically ten to a hundred times fainter than the SDSS AGNs. The Palomar LLAGNs show a different behavior to the SDSS AGNs: the numerous Palomar LLAGNs with low black hole masses are accreting a similar mass per year as the fewer Palomar LLAGNs and Palomar AGNs at higher radio luminosity. This is true whether the accretion rate is calculated from the [O III] luminosity, as in Heckman et al. (2004), or from $Q_{\text {jet }}$ (e.g. Fig. 5, upper $x$-axis).

The Palomar sample ellipticals (filled symbols in Fig. 10) show a strong correlation between Eddington ratio, $l_{\text {Edd }}$ (calculated assuming the jet power dominates the accretion energy output) and all of emission-line luminosity, radio luminosity, and the ratio of radio luminosity to emission line luminosity. Among the non-elliptical nuclei (open symbols in Fig. 10), Seyfert nuclei (triangles) display higher Eddington ratios than LINERs (circles), even though the distribution of radio luminosities (from which $Q_{\text {jet }}$ is calculated) is similar for the two classes. This is most clearly noticeable in Fig. 10c.

\subsection{Discovery of LLAGNs: A comparison of radio, optical, and $X$-ray methods}

The current study and sample allows a comparison of the relative success of deep optical spectroscopy, high resolution radio imaging, and (to some extent) hard X-ray imaging, in identifying low-luminosity accreting black holes. Such a comparison is specially relevant in view of the several hundred thousand AGNs and LLAGNs being identified by current large surveys out to $z \sim 6$. We consider three factors here: efficiency, reliability, and completeness. In this subsection, we shall use the term "AGN" to mean "AGN + LLAGN", namely objects powered by accretion onto a supermassive black hole.

The optical spectroscopy (of 486 nuclei) was obtained at the $5 \mathrm{~m}$ Hale telescope with typical exposures of $30 \mathrm{~min}$ to $1 \mathrm{hr}$ per nucleus. Has this optical spectroscopy missed any (radioor X-ray identified) AGNs in the Palomar sample? The spectroscopic survey found emission-lines in all except 53 nuclei (Ho et al. 2003a). Of these 53 absorption line nuclei we found only 


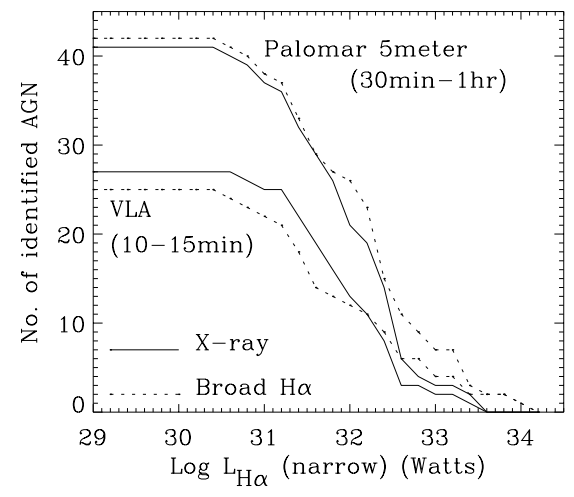

Fig. 11. The cumulative number of "definite" (solid lines for nuclei with a hard X-ray nuclear source and dashed lines for type 1 nuclei, i.e. with broad $\mathrm{H} \alpha$ emission) AGNs identified by optical spectroscopy (upper two lines; $30 \mathrm{~min}$ to $1 \mathrm{~h}$ on the Palomar $5 \mathrm{~m}$ ) and radio imaging (lower two lines; $\sim 10-15 \mathrm{~min}$ at the VLA), as a function of the luminosity of the narrow $\mathrm{H} \alpha$ line.

two with radio nuclei which would identify them as definite AGNs, and a further three to five with radio nuclei possibly related to an AGN (see the appendix). We could not find reliable hard X-ray identifications of AGNs, for lack of data, in any of the absorption line nuclei. The H II nuclei in the Palomar sample also show no indication of an AGN in the radio (Ulvestad \& Ho 2001b) or X-ray. Thus, the optical spectroscopic survey has missed only two definite (and perhaps three or five probable) AGNs which would have been picked up by a radio survey with detection limit $\sim 1 \mathrm{mJy}$. The reliability of AGN identification from optical spectroscopy alone is hard to quantify. At these low luminosities, emission lines could be powered by sources other than an AGN, e.g. a nuclear starburst (Maoz et al. 1998).

The radio imaging (of $\sim 200$ nuclei) was done with the VLA with typical integration times of 10 to $15 \mathrm{~min}$ per nucleus. Follow-up VLBA imaging ( $1 \mathrm{~h}$ per nucleus) showed that the VLA-only imaging could be used for reliable identification of the radio nuclei as AGN-related (Sect. 4.2). The high brightness temperature radio nuclei and parsec-scale jets, found through VLBA observations, are the most reliable indicators of AGNs in these nuclei as a class. Our radio imaging has identified fewer AGN candidates than the optical spectroscopy, though it is likely that deeper radio imaging will uncover significantly more AGNs (Nagar et al. 2002a). It is difficult to ascertain whether the radio survey has missed any (definite) optically identified AGNs, since the detection of weak emission lines does not guarantee the presence of a low-luminosity accretionpowered nucleus. Ideally, one requires hard X-ray confirmation of the presence of an AGN: this issue will be more fully discussed in Terashima et al. (in prep.). The cumulative number of "definite" AGNs identified by the optical spectroscopic and the radio imaging methods, as a function of the luminosity in the narrow $\mathrm{H} \alpha$ line, is shown in Fig. 11. Here we use the presence of either a nuclear hard X-ray source (solid lines; Fig. 11) or broad $\mathrm{H} \alpha$ emission (dashed lines; Fig. 11) as the signpost of a "definite" AGN - i.e. an object powered by accretion onto a supermassive black hole. Our radio survey has detected 26 of $39(66 \%)$ of the type 1 nuclei in the sample. Of the 14 type 1 nuclei not detected in our radio survey, all
5 Seyferts were found to have radio nuclei in the deeper radio survey of Ho \& Ulvestad (2001), while the nature of the others is unknown.

In summary, optical spectroscopy of the Palomar sample has found almost all radio or X-ray identified AGNs to have emission-lines: as discussed in the Appendix, very few of the nuclei with only absorption lines have radio emission likely powered by an accreting black hole. A caveat here is that the absorption line nuclei have not been surveyed in precisely the same way as we have surveyed the AGNs and LLAGNs. The optical spectroscopic method also finds many nuclei with emission lines powered by hot stars (H II nuclei) and these emission lines can hide weaker emission lines from an AGN. High resolution, high frequency radio imaging (the present survey) has detected a smaller fraction of AGN candidates in the Palomar sample than the optical spectroscopic method, but we have argued that these radio sources are not related to stellar processes. Thus, the presence of a compact flat-spectrum high brightness-temperature radio core is a more reliable indicator of an accreting black hole than the presence of optical emission lines, at least at these low emission line and radio luminosities. A hard X-ray nucleus is also an ideal signpost of an AGN. However, high resolution (i.e. Chandra) is required to minimize confusion with X-ray binaries or Ultraluminous X-ray sources (ULXs), which have similar luminosities to the AGN in LLAGNs. Further, a large X-ray survey is very expensive in terms of telescope time. Instead, scientific results can be efficiently attained by hard X-ray observations of subsamples selected to have a compact radio core (Terashima \& Wilson 2003; Terashima et al., in prep).

\section{Discussion}

The VLBI results (Sect. 4.2 and Table 2) confirm that almost all (38 of 39, or 97\%) LLAGNs and AGNs in the Palomar sample with $S_{15 \mathrm{GHz}}^{\mathrm{VLA}}>2.7 \mathrm{mJy}$ have detected mas-scale or sub masscale radio nuclei with brightness-temperature $\gtrsim 10^{7} \mathrm{~K}$. The only exception is NGC 2655: this nucleus has a steep spectrum at arcsec resolution (Nagar et al. 2000) and was not detected by us with the VLBA. Deeper VLBA/I maps show mas-scale radio nuclei in five Palomar LLAGNs with $S_{15 \mathrm{GHz}}^{\mathrm{VLA}} \leq 2.7 \mathrm{mJy}$ (Table 2). It is notable that the LLAGNs and AGNs with known ultracompact radio nuclei are divided between Seyferts and LINERs in proportion to their relative numbers in the Palomar sample (14 and 23, respectively; see Table 2). Thus the probability of detecting ultracompact radio nuclei in LLAGNs with Seyfert and LINER spectra is similar. Nuclear starbursts have a maximum brightness-temperature of $\sim 10^{4-5} \mathrm{~K}$ (Condon et al. 1991) while the most luminous known radio supernova remnants (e.g. Colina et al. 2001) would have brightness temperatures $\leq 10^{7} \mathrm{~K}$ even if they were $\leq 1 \mathrm{pc}$ in extent. As argued in Falcke et al. (2000), if the nuclear radio emission is attributed to thermal processes, the predicted soft X-ray luminosities of LLAGNs would be at least two orders of magnitude higher than observed by ASCA (Terashima et al. 2000) and Chandra (Ho et al. 2001; Terashima \& Wilson 2003; Filho et al. 2004). Also, as pointed out by Ulvestad \& Ho (2001a), single SNRs (Colina et al. 2001) or a collection of SNRs 
(Neff \& Ulvestad 2000) would have radio spectral indices (defined by $\left.S \propto v^{\alpha}\right) \alpha \sim-0.7$ to -0.4 rather than the values $\alpha \sim-0.2$ to 0.2 seen in the VLBA-detected LLAGNs (Nagar et al. 2001, 2002b; Anderson et al. 2004; Nagar et al., in prep.) Furthermore, significant flux variability is observed (Nagar et al. 2002a). Thus, the only currently accepted paradigm which may account for the sub-parsec radio nuclei is accretion onto a supermassive black hole. In this case, the mas-scale radio emission is likely to be either emission from the accretion inflow (Narayan et al. 2000) or synchrotron emission from the base of the radio jet launched by the accreting supermassive black hole (Falcke \& Biermann 1999; Zensus 1997). The latter model is supported by the presence of sub-parsec size jets in many of the nuclei, and the radio spectral shape (Nagar et al. 2001, 2002a,b; Anderson et al. 2004).

The radio results imply that a large fraction (perhaps all) of LLAGNs have accreting massive black holes. If we consider only the detections of mas-scale radio sources, then at least $25 \% \pm 5 \%$ of LINERs and low-luminosity Seyferts have accreting black holes. VLA-detected compact radio nuclei with flux $<2.7 \mathrm{mJy}$ were not investigated with the VLBA; in other respects these nuclei are similar to those with detected mas scale structure. Thus it is likely that all LLAGNs with VLAdetected compact radio nuclei $(42 \% \pm 7 \%$ of LINERs and lowluminosity Seyferts) have accreting black holes. The scalings between radio luminosity, emission-line luminosity, and galaxy luminosity (Ulvestad \& Ho 2001a; Nagar et al. 2002a; Filho et al. 2004) provide evidence that the radio non-detections are simply lower luminosity versions of the radio detections. In fact we find no reason to disbelieve that all LLAGNs have an accreting black hole.

Interestingly, ultracompact radio nuclei (Table 2 ) are found almost exclusively in massive $\left(M_{\mathrm{B}}\right.$ (total $\left.) \leq-20\right)$ ellipticals and in type 1 LLAGNs, or both. For massive ellipticals, the high bulge luminosity and black hole mass appear to be key factors related to the production of a radio nucleus, in light of the scalings seen between radio luminosity and these parameters (see Fig. 4 of present paper and Nagar et al. 2002a). Among non-ellipticals, the preferential detection of type 1 LLAGNs may result from the limited sensitivity of optical and radio observations, which detect broad $\mathrm{H} \alpha$ and radio nuclei in only the more luminous LLAGNs. For example, it may be that type 1 LLAGNs are in an outburst phase during which they temporarily host both broad $\mathrm{H} \alpha$ emission and a compact radio nucleus. Type 2 LLAGNs, on the other hand, may harbor quiescent AGNs which do not generate sufficient ionizing photons to power the optical emission lines (e.g. Terashima et al. 2000; Filho et al. 2004). Instead, their emission lines could be powered by star formation related processes (Maoz et al. 1998). As another alternative, one can invoke the unified scheme (Antonucci 1993) and posit that all LLAGNs have accreting black holes and either (a) the radio emission in type 1 LLAGNs is beamed (weakly relativistic jets $[\gamma \sim 2]$ can give boost factors of up to $\sim 5$ ) and/or (b) the $15 \mathrm{GHz}$ radio emission in type 2 LLAGNs is free-free absorbed by a "torus"-like structure i.e. $\tau_{15 \mathrm{GHz}} \geq 1$.

The radio and emission line properties of LLAGNs in elliptical galaxies are consistent with them being scaled-down
FR Is (Sects. 5.1 and 5.5), confirming earlier such suggestions with smaller samples (Nagar et al. 2002a; Verdoes Kleijn et al. 2002; Chiaberge et al., in prep.). Additionally, in the context of jet models, the same scaling relationship between jet kinetic power and radiated NLR luminosity is followed by parsec-scale jets in LLAGNs as kpc-scale jets in powerful FR I and FR II radio galaxies (Fig. 8 and Sect. 5.5).

The nuclear environments of low-luminosity Palomar Seyferts are richer in gas than those of Palomar LINERs (Ho et al. 2003a), as inferred from higher electron densities $\left(n_{\mathrm{e}}\right)$ and higher internal extinction in the former class. We have found that among non-elliptical hosts, LINER nuclei have lower Eddington ratios than Seyfert nuclei (Fig. 10 and Sect. 5.6). Also, we find evidence for a higher incidence of parsec-scale radio jets in LINERs than Seyferts (Sect. 5.4); at least some low luminosity Seyferts do show larger (100 pc scale) jets. It is tempting to speculate, in analogy to Galactic black hole candidates (Fender \& Belloni 2004), that LINERs with radio nuclei are in a "low/hard" state (low Eddington ratio, lack of inner accretion disk, more efficient at launching collimated jets) while low-luminosity Seyferts are in a "high" state (higher Eddington ratios, less efficient at launching collimated jets).

Luminous Seyferts, the Palomar sample, and the local group of galaxies together allow an estimate of the nuclear radio luminosity function over the radio luminosity range $10^{15}-10^{24}$ Watt $\mathrm{Hz}^{-1}$, more than five orders of magnitude larger than previous AGN samples. At the lowest luminosities there is tentative evidence for a turnover in the RLF. One must therefore reach as far down as the LLAGN regime (but not necessarily lower) to completely study the demographics of nuclear accretion. This point is especially important since larger surveys, e.g. SDSS, probe accretion in AGNs with $l_{\text {Edd }}$ one or two orders of magnitude larger than that in the Palomar sample LLAGNs.

When only the radiated luminosity is considered, LLAGNs have very low inferred Eddington ratios. This requires either a very low mass accretion rate or a radiatively inefficient accretion mechanism or both, and was among the original motivations for invoking RIAFs in LLAGNs (but see Binney 2004a, for an argument against the existence of RIAFs). Including the jet power (Sect. 5.5) in the accretion output weakens the motivation for a RIAF. First, including the jet kinetic power significantly increases the total (radiated plus kinetic) $L_{\text {Emitted }}$ and thus the Eddington ratio. Second, the energy deposited by the jet into the nuclear regions can potentially heat the gas in the inner parsecs and thus decrease gas supply to the accretion disk (Sect. 5.5.3). Together these two factors weaken the previous preference for RIAFs over a matter-starved accretion disk plus jet system. The absence in LLAGNs of the "big blue bump" and $\mathrm{Fe} \mathrm{K} \alpha$ lines are thus the main remaining motivations for preferring an optically-thin, geometrically thick and advection dominated inner accretion structure over the standard opticallythick, geometrically thin accretion disk.

Jet models indicate that the dominant form of power output in LLAGNs is the kinetic power of the jet (Sect. 5.5). The Eddington ratios found are $l_{\text {Edd }} \sim 10^{-2}$ to $10^{-6}$ (Figs. 4, 7 and 10). In terms of a mass accretion rate (assuming a $10 \%$ conversion efficiency) this translates to $\dot{M}=10^{-1}$ to $10^{-5} M_{\odot} \mathrm{yr}^{-1}$ 
(upper $x$-axis of Fig. 5). The summed mass accretion rate for all Palomar LLAGNs and AGNs in Fig. 5 is $0.4 M_{\odot} \mathrm{yr}^{-1}$. Now, the idealised jet model of Eq. (20) of Falcke \& Biermann (1999) predicts $\log Q_{\text {jet }} \propto 0.79 \times \log L_{v}$ (where $L_{v}$ is the observed luminosity at radio frequency $v$ ) for a jet inclination of $45^{\circ}$, while the Palomar RLF has the form $\log \rho \propto-0.78 \times \log L_{15 \mathrm{GHz}}$. While there are large uncertainties in applying the jet model, it is worth remarking that these scalings imply $Q_{\text {jet }} \propto \rho^{-1}$. This implies (since $Q_{\text {jet }} \propto \dot{M}$ under the assumption that the jet power dominates the accretion power output) that the more numerous nuclei with lower radio luminosity are together accreting the same mass per year as the fewer nuclei at higher radio luminosity, at least down to the probable RLF turnover at $\log L_{15 \mathrm{GHz}} \simeq 19 \mathrm{Watt}^{-1}$. Finally, with individual jet powers of $\sim 10^{40}-10^{45} \mathrm{erg} \mathrm{s}^{-1}$, LLAGN jets provide a significant source of energy (Sect. 5.5) into the galactic ISM and also perhaps the IGM. As discussed in Di Matteo et al. (2001) a fraction of this jet power deposited within the central $\sim 0.1 \mathrm{kpc}$ would be sufficient to significantly lower the accretion rate at least in the case of spherical accretion.

\section{Conclusions}

We have presented the results of our VLA plus VLBA radio survey of 162 LLAGNs and AGNs from the Palomar sample of nearby bright northern galaxies. These data have been supplemented by data and results from two other recent surveys, one by Ho \& Ulvestad (2001), Ulvestad \& Ho (2001a) and Anderson et al. (2004) and the other by Filho et al. (2000) and Filho et al. (2004). The completion of uniform high resolution radio surveys of the LLAGNs and AGNs in the Palomar sample of nearby bright galaxies has yielded the following main results:

a) $97 \%$ (38 of 39) of the LLAGNs and AGNs (with $S_{\mathrm{VLA}}^{15 \mathrm{GHz}}>$ $2.7 \mathrm{mJy}$ ) investigated at mas resolution with the VLBA have pc-scale nuclei with brightness temperatures $\gtrsim 10^{6.3} \mathrm{~K}$. Of these nuclei, the ones with the highest nuclear flux densities typically show pc-scale jets. The luminosity, brightness temperature, spectrum, morphology, and variability of the radio emission all argue against an origin in starformation related processes or as thermal emission. Thus, the nuclear radio emission probably originates either in an accretion inflow onto a supermassive black hole or from jets launched by this black hole-accretion disk system. The latter explanation is supported by the radio morphologies (e.g. Falcke et al. 2000; Nagar et al. 2002a), radio flux variability (Nagar et al. 2002a), and the radio spectral shapes (Nagar et al. 2001, 2002b);

b) there is no reason to believe that the remaining LLAGNs with compact radio nuclei (investigated at 150 mas resolution) are different from the LLAGNs investigated at mas resolution. Thus, at least half of all LINERs and lowluminosity Seyferts probably contain accreting black holes. The incidence for transition nuclei is much lower;

c) compact radio nuclei are preferentially found in massive ellipticals and in type 1 nuclei (i.e. nuclei in which broad $\mathrm{H} \alpha$ emission is present). The preferential detection of type 1 nuclei could result: 1) from observational selection effects, in which broad $\mathrm{H} \alpha$ and radio nuclei have been found in only the more powerful LLAGNs; 2) if only the type 1 LLAGNs are bona-fide AGNs; or 3) if the unified scheme applies and the radio emission from type 1 nuclei is beamed perpendicular to the plane of obscuring material and/or type 2 nuclei are free-free absorbed by the obscuring disk in the radio;

d) the radio luminosity of the compact nucleus is correlated with the galaxy luminosity and the luminosity and width of the nuclear emission lines (Nagar et al. 2002a). These trends suggest that we have detected only the brighter LLAGNs, i.e. the true incidence of accreting black holes in LLAGNs is higher than found by our survey;

e) The nuclear radio and nuclear emission-line properties of LLAGNs fall close to the low-luminosity extrapolations of more powerful AGNs, providing further support for a common central engine;

f) low-luminosity Seyferts and LINERs share many of the same characteristics in the radio. The transition nuclei detected are those which are the closest, in terms of emissionline diagnostic ratios, to Seyferts and LINERs (Nagar et al. 2002a). Thus at least some transition nuclei are really composite Seyfert/LINER + H II region nuclei, with the nuclear radio luminosity dependent on the Seyfert/LINER component;

g) investigation of all $\sim 50$ nearby bright galaxies (most of them LLAGNs) with one radio component relatively unambiguously identified with the central engine at sub-parsec resolution, shows that the sub-parsec radio luminosity is correlated with both the estimated mass of the nuclear black hole and the galaxy bulge luminosity. The large scatter in radio luminosity at any given black hole mass may be caused by a range of accretion rates (Eddington ratios $\sim 10^{-1}-10^{-5}$ );

h) about half of all LLAGNs investigated show significant inter-year variability at $15 \mathrm{GHz}(2 \mathrm{~cm})$ and $8.4 \mathrm{GHz}$ $(3.6 \mathrm{~cm})$ (Nagar et al. 2002a);

i) the nuclear radio luminosity function at $15 \mathrm{GHz}$ of luminous Seyferts, the Palomar sample, and the local group of galaxies extends over the range $10^{15}-10^{24} \mathrm{Watt}_{\mathrm{Hz}}^{-1}$, more than five orders of magnitude larger than previous AGN samples. We find $\log \rho \propto-0.78 \times \log L_{15 \mathrm{GHz}}$ (where $\rho$ is the space density in $\mathrm{Mpc}^{-3} \mathrm{mag}^{-1}$ ), with some evidence for a low luminosity turnover near $\log L_{15 \mathrm{GHz}} \sim 19$;

j) within the context of jet models, the primary accretion energy output from LLAGNs with compact radio cores is jet kinetic power; this jet power could dominate the radiated bolometric luminosity by factors of $\sim 2$ to $>10^{2}$. These jets, which are energetically more significant than supernovae in the host galaxy, can potentially deposit sufficient energy into the inner parsecs to significantly slow gas flow into the accretion disk;

k) within the context of jet models, the mass accretion function for Palomar LLAGNs and AGNs has the form $\log \rho \propto$ $-1 \times \log \dot{M}$. That is, within the Palomar sample, the numerous LLAGNs with low radio luminosity are together accreting the same mass per year as the fewer LLAGNs and AGNs at higher radio luminosity; 
Table 3. Radio fluxes for Palomar absorption-line nuclei.

\begin{tabular}{|c|c|c|c|c|c|c|c|}
\hline Name & $\begin{array}{c}\mathrm{L}_{\mathrm{H} \alpha} \\
(\mathrm{erg} / \mathrm{s})\end{array}$ & $\begin{array}{l}S_{\text {radio }} \\
\text { (mJy) }\end{array}$ & Ref. & Name & $\begin{array}{c}\mathrm{L}_{\mathrm{H} \alpha} \\
(\mathrm{erg} / \mathrm{s})\end{array}$ & $\begin{array}{l}S_{\text {radio }} \\
\text { (mJy) }\end{array}$ & Ref. \\
\hline NGC 147 & $<35.19$ & $<1.5$ & NVSS & NGC 4382 & $<38.10$ & $<0.5$ & WH91 \\
\hline NGC 205 & $<34.79$ & 1.0 & N04 & NGC 4406 & $<37.82$ & $<0.5,0.6$ & WH91,KJ02 \\
\hline NGC 221 & $<36.19$ & $<0.03$ & T03 & NGC 4417 & $<37.88$ & $<0.5$ & WH91 \\
\hline NGC 507 & $<38.74$ & $1.4-100$ & G88,NVSS & NGC 4421 & $<37.37$ & $<1.5 \mathrm{mJy}$ & NVSS \\
\hline NGC 628 & $<36.69$ & $\ldots$ & $\ldots$ & NGC 4442 & $<37.98$ & $<0.5$ & WH91 \\
\hline NGC 821 & $<38.13$ & $<0.5,<1.5$ & WH91,N04 & NGC 4461 & $<37.92$ & $<0.5$ & WH91 \\
\hline NGC 1023 & $<37.82$ & $<1.5$ & N04 & NGC 4473 & $<38.06$ & $<2.0$ & WH91 \\
\hline NGC 2300 & $<38.19$ & 0.7 & F89 & NGC 4478 & $<37.67$ & $<5.0$ & WH91 \\
\hline NGC 2549 & $<38.05$ & $<0.1$ & KJ02 & NGC 4503 & $<37.67$ & $<0.5$ & WH91 \\
\hline NGC 2634 & $<38.07$ & $\ldots$ & $\ldots$ & NGC 4564 & $<37.83$ & $<0.5$ & WH91 \\
\hline NGC 2775 & $<37.94$ & $<1.5$ & H87 & NGC 4570 & $<38.02$ & $<0.5$ & WH91 \\
\hline NGC 2950 & $<38.42$ & $<1.5$ & NVSS & NGC 4578 & $<37.58$ & $<0.5$ & WH91 \\
\hline NGC 3115 & $<37.80$ & $<0.4$ & F89 & NGC 4608 & $<37.65$ & $<1.0$ & WH91 \\
\hline NGC 3384 & $<37.56$ & $<0.5$ & WH91 & NGC 4612 & $<37.63$ & $<0.5$ & WH91 \\
\hline NGC 3610 & $<38.55$ & $<0.5,1.2$ & WH91,KJ02 & NGC 4621 & $<37.99$ & $<0.5$ & WH91 \\
\hline NGC 3613 & $<38.32$ & $<0.5$ & WH91 & NGC 4638 & $<37.93$ & $<0.5$ & WH91 \\
\hline NGC 3640 & $<38.18$ & $<0.5$ & WH91 & NGC 4648 & $<38.41$ & $<0.5$ & WH91 \\
\hline NGC 3838 & $<38.12$ & $<1.5$ & NVSS & NGC 4665 & $<37.93$ & $<0.4$ & F89 \\
\hline NGC 4026 & $<38.10$ & 1.4 & WH91 & NGC 4649 & $<37.73$ & $\sim 18 \mathrm{mJy}$ & SW86 \\
\hline NGC 4179 & $<38.45$ & $<0.5$ & WH91 & NGC 4754 & $<38.01$ & $<0.5$ & WH91 \\
\hline NGC 4251 & $<37.74$ & $<0.5$ & WH91 & NGC 4914 & $<38.84$ & $<1.0$ & FIRST \\
\hline NGC 4267 & $<37.88$ & $<0.5$ & WH91 & NGC 5473 & $<38.39$ & $<0.5$ & WH91 \\
\hline NGC 4291 & $<38.38$ & $<0.4$ & WH91,F89 & NGC 5576 & $<38.49$ & $<0.1$ & WH91,KJ02 \\
\hline NGC 4339 & $<38.02$ & $<0.5$ & WH91 & NGC 6654 & $<38.11$ & $<1.5$ & NVSS \\
\hline NGC 4365 & $<37.98$ & $<0.1$ & WH91,KJ02 & NGC 7332 & $<38.03$ & $<1.5$ & N04 \\
\hline NGC 4371 & $<37.61$ & $<0.5$ & WH91 & NGC 7457 & $<37.24$ & 1.1 & N04 \\
\hline NGC 4379 & $<37.63$ & $<0.5$ & WH91 & $\ldots$ & $\ldots$ & . & $\ldots$ \\
\hline
\end{tabular}

aThis table lists all 53 nuclei without detected emission-lines in the Palomar Sample (Ho et al. 2003). The columns list the galaxy name (in bold if the nucleus has radio flux $\geq 1 \mathrm{mJy}$ ), upper limit to the $\mathrm{H} \alpha$ luminosity (from Ho et al. 2003), the VLA radio flux (at the highest resolution found; multiple values are listed when significantly different data are available), and reference(s) for the radio flux.

' The reference codes are: F89: Fabbiano et al. 1989, $5 \mathrm{GHz}, \sim 0 !^{\prime \prime} 6$ or $\sim 5^{\prime \prime}$ resolution; FIRST: VLA FIRST survey (White et al. 1997), $1.4 \mathrm{GHz}, 5^{\prime \prime}$ resolution; H87: Hummel et al. (1987), $1.4 \mathrm{GHz}, 1{ }^{\prime \prime} 5$ resolution; KJ02: Krajnović \& Jaffe (2002), 8.4 GHz, 2." 5 resolution; N04: this work, $15 \mathrm{GHz}, 150$ mas resolution; NVSS: VLA NVSS survey (Condon et al. 1998), $1.4 \mathrm{GHz}, 45^{\prime \prime}$ resolution; SW86: Stanger \& Warwick (1986), $1.4 \mathrm{GHz}$ and $5 \mathrm{GHz}$ imaging with resolution 4" and 1." 2, respectively; WH91: Wrobel \& Heeschen (1991), $5 \mathrm{GHz}, 5^{\prime \prime}$ resolution.

c Notes: NGC 507 (B2 0120+33) has twin radio lobes and a weak 1.4 mJy core; NGC 4406 was not detected by WH91, but detected by KJ02; NGC 4649 has a core plus twin jet structure.

1) with increasing Eddington ratio, $l_{\text {Edd }}$, LLAGNs in elliptical galaxies are increasingly radio-loud as measured by the ratio of radio to optical emission-line luminosities;

m) among non-elliptical hosts, LINERs have lower nuclear gas densities, lower Eddington ratios, and are more efficient at launching collimated sub-parsec jets, than low-luminosity Seyferts. We speculate that, by analogy with Galactic black hole candidates, LINERs are in a "low/hard" state while Seyferts are in a "high" state (Sect. 6);

n) high resolution radio imaging is an effective and efficient search technique for finding low luminosity accreting black holes in LLAGNs.

In short, all evidence points toward the presence of accreting black holes in a large fraction, perhaps all, of LLAGNs. Compact radio jets are an energetically important product of accretion in this low luminosity regime. The radio luminosity function and the jet kinetic powers of these LLAGNs together show that jets are the dominant form of power output from the nuclear accretion and suggest that the jets may input a significant amount of energy into the ISM of their host galaxies.
Acknowledgements. This work was partly funded by the Dutch research organization NWO, through a VENI grant to NN, and by NASA through grants NAG513065 and NAG513557 to the University of Maryland. Parts of this work were completed while NN held a fellowship at Arcetri Observatory, Italy.

\section{Appendix: Absorption-line nuclei in the Palomar sample}

The Palomar spectroscopic survey did not detect emission lines in 53 nuclei of the Palomar Sample; upper limits to the $\mathrm{H} \alpha$ luminosity in these nuclei are listed in Ho et al. (2003a) ${ }^{5}$. We have searched the literature and VLA archive for high resolution radio observations of these 53 nuclei. The results are listed in Table 3. Only two of the nuclei have AGN-related radio fluxes significantly greater than $\sim 1 \mathrm{mJy}$. NGC 507 (also known as B2 0120+33) has about $100 \mathrm{mJy}$ at $1.4 \mathrm{GHz}$ in larger

\footnotetext{
${ }^{5}$ Ho et al. (2003a) list 54 nuclei with $\mathrm{H} \alpha$ luminosity upper limits; of these, the nucleus of NGC 4494 shows other emission lines and was classified as a (highly uncertain) "LINER 2" by Ho et al. (1997a).
} 
scale radio lobes but a very weak $(\sim 1.4 \mathrm{mJy}$ at $5 \mathrm{GHz})$ radio nucleus (Giovannini et al. 1988); it may be that the AGN is now switched off or in a very low state, which could account for the lack of emission-lines. NGC 4649 has a $1.4 \mathrm{GHz}$ flux of $18 \mathrm{mJy}$ which is distributed in a core plus twin jet structure (Stanger \& Warwick 1986). Another three (possibly 5) of the 53 nuclei have weak $(\sim 1 \mathrm{mJy})$ radio nuclei, potentially AGNrelated, at $1-5^{\prime \prime}$ resolution (Table 3 ).

\section{References}

Alonso-Herrero, A., Rieke, M. J., Rieke, G. H., \& Shields, J. C. 2000, ApJ, 530, 688

Anderson, J. M., Ulvestad, J. S., \& Ho, L. C. 2004, ApJ, 603, 42

Antonucci, R. R. J. 1993, ARA\&A, 31, 473

Barth, A. J., Filippenko, A. V., \& Moran, E. C. 1999, ApJ, 525, 673

Barth, A. J., Ho, L. C., Filippenko, A. V., \& Sargent, W. L. W. 1998, ApJ, 496, 133

Beasley, A. J., Gordon, D., Peck, A. B., et al. 2002, ApJS, 141, 13

Begelman, M. C., Blandford, R. D., \& Rees, M. J. 1984, Rev. Mod. Phys., 56, 255

Bietenholz, M. F., Bartel, N., \& Rupen, M. P. 2000, ApJ, 532, 895

Binney, J. 2004a, MNRAS, to appear [arXiv: astro-ph/0308171]

Binney, J. 2004b, to appear in Phil. Trans. Roy. Soc. [arXiv: astro-ph/0407238]

Binney, J., \& Tremaine, S. 1987, Galactic Dynamics (Princeton, NJ: Princeton University Press), 747

Blandford, R. D. 1993, in Astrophysical Jets, ed. D. Burgarella, M. Livio, \& C. P. O'Dea, (Cambridge: Cambridge Univ. Press), 15

Braatz, J., Wilson, A. S., \& Henkel, C. 1997, ApJS, 110, 321

Carral, P., Turner, J. L., \& Ho, P. T. P. 1990, ApJ, 362, 434

Celotti, A., \& Fabian, A. C. 1993, MNRAS, 264, 228

Chatterjee, S., Cordes, J. M., Vlemmings, W. H. T., et al. 2004, ApJ, 604, 339

Colina, L., Alberdi, A., Torrelles, J. M., Panagia, N., \& Wilson, A. S. 2001, ApJ, 553, L19

Condon, J. J. 1989, ApJ, 338, 13

Condon, J. J., Huang, Z.-P., Yin, Q. F., \& Thuan, T. X. 1991, ApJ, 378, 65

Condon, J. J., Cotton, W. D., Greisen, E. W., et al. 1998, AJ, 115, 1693 Cotton, W. D., Condon, J. J., \& Arbizzani, E. 1999a, ApJS, 125, 409

Cotton, W. D., Feretti, L., Giovannini, G., Lara, L., \& Venturi, T. 1999b, ApJ, 519, 108

Crane, P. C., Cowan, J. J., Dickel, J. R., \& Roberts, D. A. 1993, ApJ, 417, L61

Dhawan, V., Kellerman, K. I., \& Romney, J. D. 1998, ApJ, 498, L111

Di Matteo, T., Carilli, C. L., \& Fabian, A. C. 2001, ApJ, 547, 731

Dopita, M. A., \& Sutherland, R. S. 1995, ApJ, 455, 468

Emsellem, E., Dejonghe, H., \& Bacon, R. 1999, MNRAS, 303, 495

Fabbiano, G., Gioia, I. M., \& Trinchieri, G. 1989, ApJ, 374, 127

Falcke, H., \& Biermann, P. L. 1999, A\&A, 342, 49

Falcke, H., Nagar, N. M., Wilson, A. S., \& Ulvestad, J. S. 2000, ApJ, 542, 197 (Paper II)

Falcke, H., Körding, E., \& Markoff, S. 2004, A\&A, 414, 895

Fender, R., \& Belloni, T. 2004, ARA\&A, 42, 317

Fender, R. P., Gallo, E., \& Jonker, P. G. 2003, MNRAS, 343, L99

Ferrarese, L., \& Merritt, D. 2000, ApJ, 539, L9

Filho, M. E., Barthel, P. D., \& Ho, L. C. 2000, ApJS, 129, 93

Filho, M. E., Barthel, P. D., \& Ho, L. C. 2002, A\&A, 385, 425

Filho, M. E., Fraternali, F., Markoff, et al. 2004, A\&A, 418, 429

Filippenko, A. V., \& Ho, L. C. 2003, ApJ, 588, L13
Filippenko, A. V., \& Terlevich, R. 1992, ApJ, 397, L79

Fomalont, E. B., Frey, S., Paragi, Z., et al. 2000, ApJS, 131, 95

Fosbury, R. A. E., Mebold, U., Goss, W. M., \& Dopita, M. A. 1978, MNRAS, 183, 549

Franceschini, A., Vercellone, S., \& Fabian, A. C. 1998, MNRAS, 297, 817

Frank, J., King, A., \& Raine, D. 1995, in Accretion Power in Astrophysics, 2nd edition, (Cambridge: Cambridge Univ. Press)

Gebhardt, K., Bender, R., Bower, G., et al. 2000, ApJ, 539, L13

Gebhardt, K., Richstone, D., Tremaine, S., et al. 2003, ApJ, 583, 92

Giovannini, G., Feretti, L., Gregorini, L., \& Parma, P. 1988, A\&A, 199,73

Giovannini, G., Cotton, W. D., Feretti, L., Lara, L., \& Venturi, T. 2001, ApJ, 552, 508

Heckman, T. M. 1980, A\&A, 87, 152

Heckman, T. M., Crane, P. C., \& Balick, B. 1980, A\&AS, 40, 295

Heckman, T. M., Kauffmann, G., Brinchmann, J., et al. 2004, ApJ, 613, 109

Herrnstein, J. R., Moran, J. M., Greenhill, L. J., et al. 1997, ApJ, 475, L17

Ho, L. C. 1999, ApJ, 516, 672

Ho, L. C. 2002, ApJ, 564, 120

Ho, L. C., \& Ulvestad, J. S. 2001, ApJS, 133, 77

Ho, L. C., Filippenko, A. V., \& Sargent, W. L. W. 1995, ApJS, 98, 477

Ho, L. C., Filippenko, A. V., \& Sargent, W. L. W. 1997a, ApJS, 112, 315

Ho, L. C., Filippenko, A. V., \& Sargent, W. L. W., \& Peng, C. Y. 1997b, ApJS, 112, 391

Ho, L. C., Feigelson, E. D., Townsley, L. K., et al. 2001, ApJ, 549, L51

Ho, L. C., Filippenko, A. V., \& Sargent, W. L. W. 2003a, ApJ, 583, 159

Ho, L. C., Terashima, Y., \& Ulvestad, J. S. 2003b, ApJ, 589, 783

Hummel, E. 1980, A\&AS, 41, 151

Hummel, E., Fanti, C., Parma, P., \& Schilizzi, R. T. 1982, A\&A, 114, 400

Hummel, E., van der Hulst, J. M., Keel, W. C., \& Kennicutt, R. C. 1987, A\&AS, 70, 517

Jones, D. L., \& Wehrle, A. E. 1997, ApJ, 484, 186

Jones, D. L., Wehrle, A. E., Piner, B. G., \& Meier, D. L. 2001, ApJ, 553, 968

Jones, D. L., Terzian, Y., \& Sramek, R. A. 1981, ApJ, 246, 28

Jones, D. L., Wrobel, J. M., \& Shaffer, D. B. 1984, ApJ, 276, 480

Junor, W., \& Biretta, J. A. 1995, AJ, 109, 500

Karachentsev, I. D., Makarov, D. I., \& Huchtmeier, W. K. 1999, A\&AS, 139, 97

Kellermann, K. I., Vermeulen, R. C., Zensus, J. A., \& Cohen, M. H. 1998, AJ, 115, 1295

Koski, A. T., \& Osterbrock, D. E. 1976, ApJ, 203, L49

Krajnović, D., \& Jaffe, W. 2002, A\&A, 390, 423

Krichbaum, T. B., Graham, D. A., Witzel, S., et al. 1998, A\&A, 335, L106

Kukula, M. J., Ghosh, T., Pedlar, A., \& Schilizzi, R. T. 1999, ApJ, 518,117

Kukula, M. J., Pedlar, A., Baum, S. A., \& O’Dea, C. P. 1995, MNRAS, 276,1262

Laurent-Muehleisen, S. A., Kollgaard, R. I., Ryan, P. J., et al. 1997, A\&AS, 122, 235

Lavalley, M., Isobe, T., \& Feigelson, E. 1992, in Astronomical Data Analysis Software and Systems I, ed. D. Worrall, C. Biemesderfer \& J. Barnes, (San Francisco: ASP), 25, 245 (ASURV)

Lonsdale, C. J., Lonsdale, C. J., \& Smith, H. E. 1992, ApJ, 391, 629

Lonsdale, C. J., Smith, H. J., \& Lonsdale, C. J. 1993, ApJ, 405, L9 
Lovelace, R. V. E., \& Romanova, M. M. 1996, in Energy Transport in Radio Galaxies, ed. P. E. Hardee, A. H. Bridle, \& J. A. Zensus (San Francisco: ASP), 100, 25

Maoz, D., Filippenko, A. V., Ho, L. C., et al. 1995, ApJ, 440, 91

Maoz, D., Koratkar, A., Shields, J. C., et al. 1998, AJ, 116, 55

Marconi, A., \& Hunt, L. K. 2003, ApJ, 589, L21

Merloni, A., Heinz, S., \& di Matteo, T. 2003, MNRAS, 345, 1057

Merritt, D., \& Ferrarese, L. 2001, MNRAS, 320, L30

Meurs, E. J. A., \& Wilson, A. S. 1984, A\&A, 136, 206

Middelberg, E., Roy, A. L., Nagar, N. M., et al. 2004, A\&A, 417, 925

Mundell, C. G., Wilson, A. S., Ulvestad, J. S., \& Roy, A. L. 2000, ApJ, 529,816

Mundell, C. G., Wrobel, J. M., Pedlar, A., \& Gallimore, J. F. 2003, ApJ, 583, 192

Nagar, N. M. 2003, to appear in the proceedings of Multiwavelength AGN Surveys, Cozumel, December 2003, ed. R. Maiolino, \& R. Mujica (World Scientific)

Nagar, N. M., Wilson, A. S., Mulchaey, J. S., \& Gallimore, J. F. 1999, ApJS, 120, 209

Nagar, N. M., Falcke, H., Wilson, A. S., \& Ho, L. C. 2000, ApJ, 542, 186 (Paper I)

Nagar, N. M., Wilson, A. S., \& Falcke, H. 2001, ApJ, 559, L87

Nagar, N. M., Falcke, H., Wilson, A. S., \& Ulvestad, J. S. 2002a, A\&A, 392, 53

Nagar, N. M., Wilson, A. S., Falcke, H., Ulvestad, J. S., \& Mundell, C. G. 2002b, Issues in Unification of Active Galactic Nuclei, ASP Conf. Ser., 258, 171

Napier, P. J., Bagri, D. S., Clark, B. G., et al. 1994, Proc. IEEE, 82, 658

Narayan, R., Mahadevan, R., \& Quataert, E. 1998, in The Theory of Black Hole Accretion Discs, ed. M. A. Abramowicz, G. Björnsson, \& J. E. Pringle (Cambridge: Cambridge Univ. Press), 148

Narayan, R., Igumenshchev, I. V., \& Abramowicz, M. A. 2000, ApJ, 539, 798

Neff, S. G., \& Ulvestad, J. S. 2000, AJ, 120, 670

Osterbrock, D. E. 1981, ApJ, 249, 462

Osterbrock, D. E. 1989, Astrophysics of Gaseous Nebulae and Active Galactic Nuclei (Mill Valley, CA: Univ. Sci. Books)

Ostriker, J. P., \& Ciotti, L. 2004, to appear in Phil. Trans. Roy. Soc. [arXiv: astro-ph/0407234]

Owen, F. N., Eilek, J. A., \& Kassim, N. E. 2000, ApJ, 543, 611

Pellegrini, S., \& Ciotti, L. 1998, A\&A, 333, 433

Pringle, J. E. 1993, in Astrophysical Jets, ed. D. Burgerella, M. Livio, \& C. P. O'Dea, (Cambridge: Cambridge Univ. Press), 1

Rawlings, S., \& Saunders, R. 1991, Nature, 349, 138

Rees, M. J., Phinney, E. S., Begelman, M. C., \& Blandford, R. D. 1982, Nature, 295, 1

Richstone, D., Ajhar, E. A., Bender, R., et al. 1998, Nature, 395A, 14

Sadler, E. M., Jenkins, C. R., \& Kotanyi, C. G. 1989, MNRAS, 240, 591

Sadler, E. M., Slee, O. B., Reynolds, J. E., \& Roy, A. L. 1995, MNRAS, 276, 1373
Sandage, A., Tammann, G. A., \& Yahil, A. 1979, ApJ, 232, 352

Sanghera, H. S., Saikia, D. J., Luedke, E., et al. 1995, A\&A, 295, 629

Schilizzi, R. T., Fanti, C., Fanti, R., \& Parma, P. 1983, A\&A, 126, 412

Shields, J. C. 1992, ApJ, 399, L27

Sjouwerman, L., Mioduszewski, A., \& Greisen, T. 2004, in preparation

Slee, O. B., Sadler, E. M., Reynolds, J. E., \& Ekers, R. D. 1994, MNRAS, 269, 928

Smith, H. E., Lonsdale, C. J., \& Lonsdale, C. J. 1998, ApJ, 492, 137

Soltan, A. 1982, MNRAS, 200, 115

Stanger, V. J., \& Warwick, R. S. 1986, MNRAS, 220, 363

Stoughton, C., Lupton, R. H., Bernardi, M., et al. 2002, AJ, 123, 485

Terashima, Y., Ho, L. C., \& Ptak, A. F. 2000, ApJ, 539, 161

Terashima, Y., \& Wilson, A. S. 2003, ApJ, 583, 145

Terlevich, R., \& Melnick J. 1985, MNRAS, 213, 841

Terlevich, R., Tenorio-Tagle, G., Franco, J., \& Melnick, J. 1995, MNRAS, 272, 198

Thompson, A. R., Clark, B. G., Wade C. M., \& Napier, P. J. 1980, ApJS, 44, 151

Tremaine, S., Gebhardt, K., Bender, R., et al. 2002, ApJ, 574, 740

Trotter, A. S., Greenhill, L. J., Moran, J. M., et al. 1998, ApJ, 495, 740

Ulvestad, J. S., \& Ho, L. C. 2001a, ApJ, 558, 561

Ulvestad, J. S., \& Ho, L. C. 2001b, ApJ, 562, L133

Ulvestad, J. S., Roy, A. L., Colbert, E. J. M., \& Wilson, A. S. 1998, ApJ, 496, 196

Ulvestad, J. S., \& Wilson, A. S. 1989, ApJ, 343, 659

Ulvestad, J. S., Wrobel, J. M., Roy, A. L., et al. 1999, ApJ, 517, L81

Venturi, T., Giovannini, G., Feretti, L., Comoretto, G., \& Wehrle, A. E. 1993, ApJ, 408, 81

van den Bergh, S., \& McClure, R. D. 1994, ApJ, 425, 205

Verdoes Kleijn, G. A., Baum, S. A., de Zeeuw, P. T., \& O’Dea, C. P. 2002, AJ, 123, 1334

Vila, M. B., Pedlar, A., Davies, R. D., Hummel, E., \& Axon, D. J. 1990, MNRAS, 242, 379

Walker, R. C., Dhawan, V., Romney, J. D., Kellermann, K. I., \& Vermeulen, R. C. 2000, ApJ, 530, 233

White, R. L., Becker, R. H., Helfand, D. J., \& Gregg, M. D. 1997, ApJ, 475, 479 (FIRST)

Woo, J., \& Urry, C. M. 2002, ApJ, 579, 530

Wrobel, J. M. 2000, ApJ, 531, 716

Wrobel, J. M., \& Heeschen, D. S. 1984, ApJ, 287, 41

Wrobel, J. M., \& Heeschen, D. S. 1991, AJ, 101, 148

Wrobel, J. M., Walker, R. C., \& Bridle, A. H. 1996, in Extragalactic radio sources: Proc. of the 175th Symposium of the IAU, ed. R. D.

Ekers, C. Fanti, \& L. Padrielli (Kluwer Academic Publishers), 131

Wrobel, J. M., Fassnacht, C. D., \& Ho, L. C. 2001, ApJ, 553, L23

Wrobel, J. M., Machalski, J., \& Condon, J. J., in preparation

Xu, C., Baum, S. A., O’Dea, C. P., Wrobel, J. M., \& Condon, J. J. 2000, AJ, 120, 2950

Zensus, J. A. 1997, ARA\&A, 35, 607

Zirbel, E. L., \& Baum, S. A. 1995, ApJ, 448, 521 